

\section{DISCLAIMER}

This report was prepared as an account of work sponsored by an agency of the United States Government. Neither the United States Government nor any agency Thereof, nor any of their employees, makes any warranty, express or implied, or assumes any legal liability or responsibility for the accuracy, completeness, or usefulness of any information, apparatus, product, or process disclosed, or represents that its use would not infringe privately owned rights. Reference herein to any specific commercial product, process, or service by trade name, trademark, manufacturer, or otherwise does not necessarily constitute or imply its endorsement, recommendation, or favoring by the United States Government or any agency thereof. The views and opinions of authors expressed herein do not necessarily state or reflect those of the United States Government or any agency thereof. 


\section{DISCLAIMER}

Portions of this document may be illegible in electronic image products. Images are produced from the best available original document. 


\title{
Tables for the Ingrowth of Daughter Products from the Decay of Plutonium Isotopes used in Heat Sources.
}

\author{
F. X. Haas and A. R. Campbel 1
}

Issued: June 12, 1972

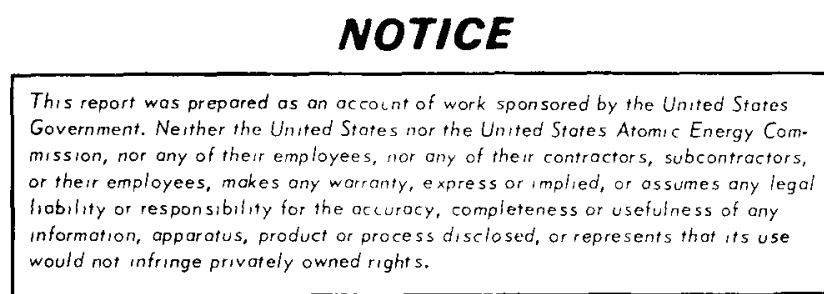

PRINTED IN THE UNITED STATES OF AMERICA Avallable from

National Technical Infornation Service

U S. Department of Commerce

5285 Port Royal Road

Springfield, Virginia 22151

Price. Printed Copy $\$ 3.00$, Microfiche $\$ 0.95$

\section{MONSANTO RESEARCH CORPORATION}

A Subsidiary of Monsanto Company

\section{MOUND LABORATORY}

Miamisburg, Onio

45342

operated for

\section{UNITED STATES ATOMIC ENERGY COMMISSION}

US Government Contract No AT-33-1-GEN-53 
page blank 


\section{TABLE OF CONTENTS}

Page

Abstract ........................ . . 4

Introduction . . . . . . . . . . . . . . . . . 5

Plutonium Decay Chains . . . . . . . . . . . . . . 5

Tables for Daughter Product Ingrowth . . . . . . . . . . 12

Use of the Tables . . . . . . . . . . . . . . . . . . 13

Tables....................... . 15

Figures. . . . . . . . . . . . . . . . . . . . . . 29

References . . . . . . . . . . . . . . . . 39 


\begin{abstract}
This paper lists in tabular and graphic form the ingrowth of activities, as a function of time, of all of the daughter products formed in the decay of the plutonium isotopes found in heat sources. The data are calculated from published half-life values using the Bateman equation. The paper is intended as a ready reference for the determination of these ingrowth data.
\end{abstract}


TABLES FOR THE INGROWTH OF DAUGHTER PRODUCTS FROM THE DECAY

OF PLUTONIUM ISOTOPES USED IN HEAT SOURCES

\section{Introduction}

This report is intended to be a quick and easy guide for determining, as a function of time, the ingrowth of atoms and total activities of the daughters of al1 plutonium isotopes present in the heat source material used at Mound Laboratory. This information has been requested repeatedly, especially for the ingrowth of ${ }^{20} \mathrm{Tl}$ from the decay of ${ }^{3{ }^{5}} \mathrm{Pu}$, for the ingrowth of ${ }^{241} \mathrm{Am}$ from ${ }^{241} \mathrm{Pu}$, and for the ingrowth of radon from all isotopes of plutonium. Hence, this document is intended as a ready reference for those persons needing such information.

\section{Plutonium Decay Chains}

The typical composition [1] of the plutonium metal used in the fabrication of the sources is shown in Table 1 .

\section{TABLE 1}

Plutonium Isotope

$$
\begin{aligned}
& 33^{6} \mathrm{Pu} \\
& 338 \mathrm{Pu} \\
& 239 \mathrm{Pu} \\
& 240 \mathrm{Pu} \\
& 341 \mathrm{Pu} \\
& 34 \text { a } \mathrm{Pu}
\end{aligned}
$$

Concentration (wt \%)

3.00

0.72

Figures $1,2,3,4,5$, and 6 show the complete decay chains for the

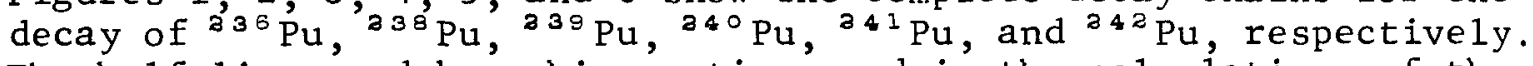
The half-lives and branching ratios used in the calculations of the ingrowth are also in the figures. These values are taken from Reference 2 unless otherwise noted. 


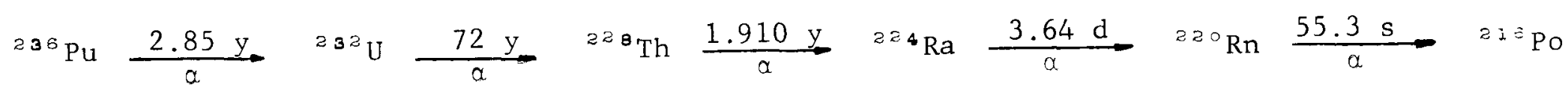

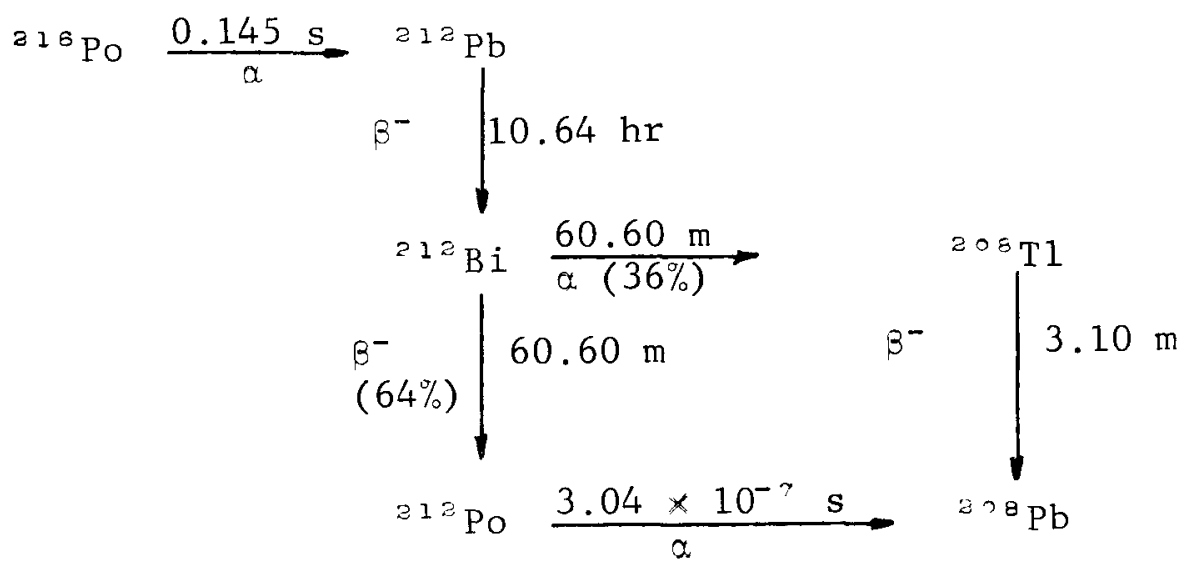

FIGURE 1 - Decay chain of ${ }^{236} \mathrm{Pu}$ 


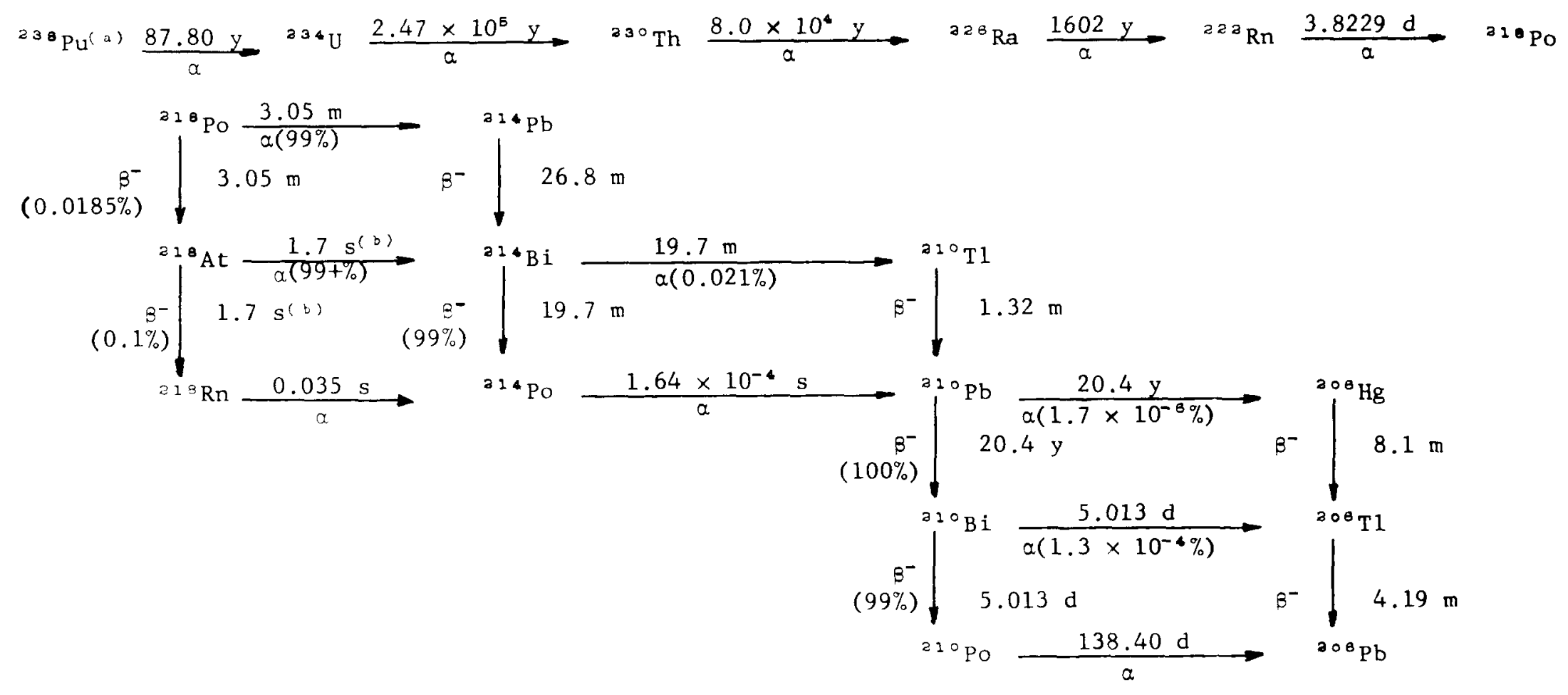

' See Reference.

(o) Intermediare vilu of range given in Referelce 2 


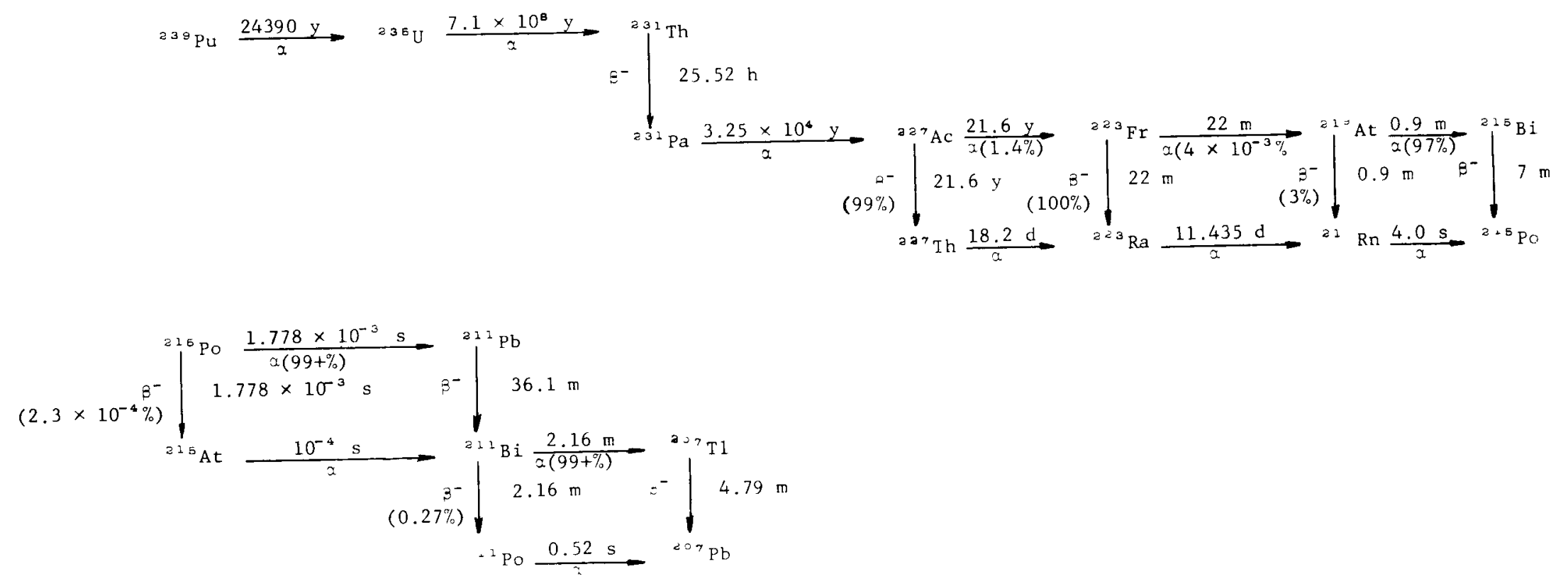

FIGURE 3 - Decay chain of $2 s^{3} \mathrm{Pu}$ 


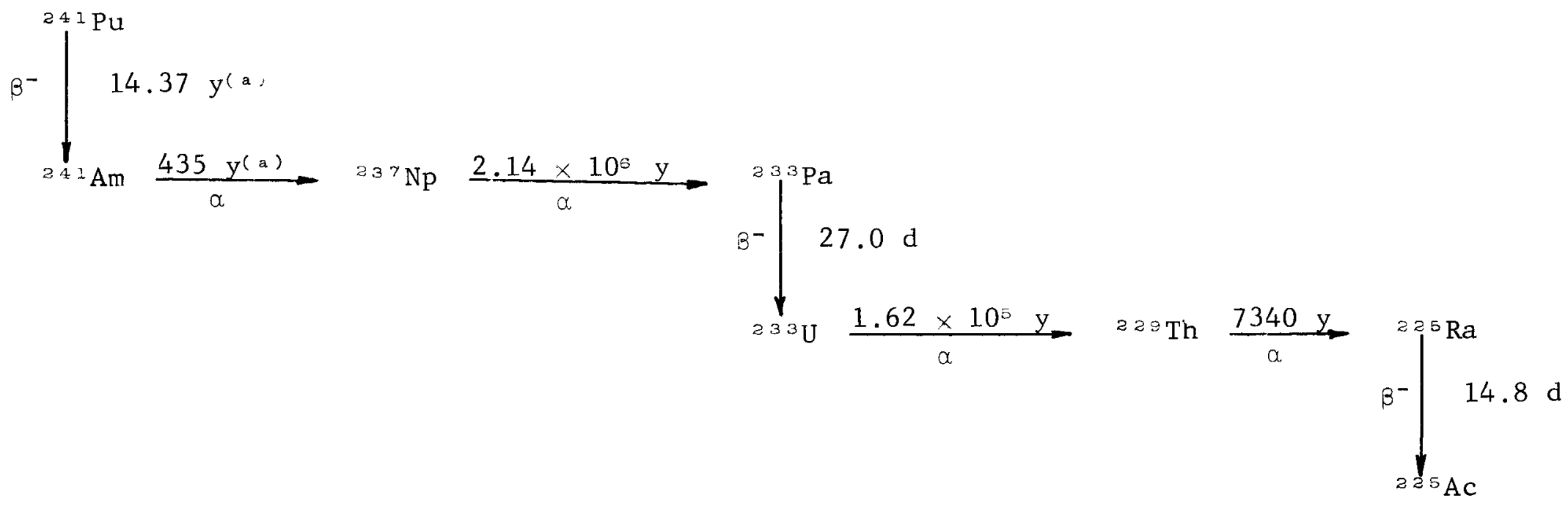




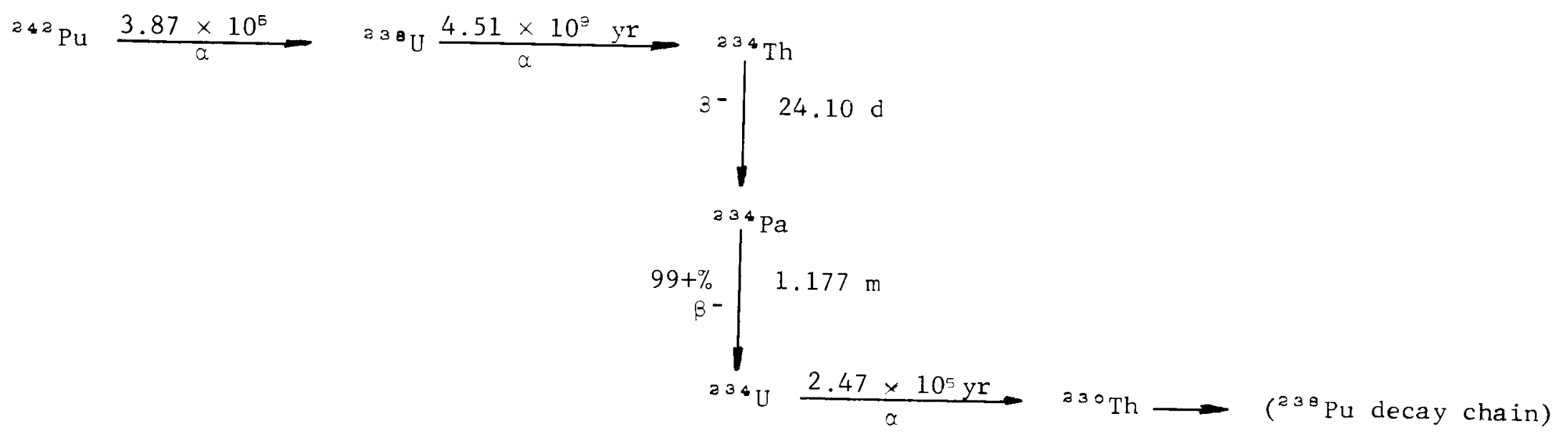


Tables for Daughter Product Ingrowth

The tables which follow list the relative number of daughter atoms and relative intensities of the daughter activities per unit value of parent plutonium isotope. The numbers in the tables were obtained from the Bateman equation for the decay of radioactive nuclide chains [ 3$\rfloor$ as programmed for a Hewlett Packard 9100B Calculator. The half-lives listed in Figures 1 through 6 were used with time conversions based on the ephemeris second (i.e., 31,556,925.9747 sec/yr and 86,400 sec/day). Calculations internal to the program were carried out with 14 digit precision. The final results were arbitrarily rounded to four significant digits even though some half-lives are not known to this accuracy. At times short compared to the half-lives involved, the terms in the Bateman equation may differ only in the 15 or greater digits in which case the 14 digit precision will give erroneous results. In such cases, those entries in the tables have been left blank. The first entry in a column can be considered as an upper limit for those blank entries in the same column.

In addition to the tables, plots of the data for the relative activities of the daughters are also included so that other times may be interpolated with ease.

The tables and figures are as follows:

Table 2: Relative Number of Atoms for Members of the ${ }^{2-5}$ Pu Decay Chain Table 3: Relative Activity for Members of the ${ }^{2}{ }^{3}$ Pu Decay Chain

Table 4: Relative Number of Atoms for Members of the $238 \mathrm{Pu}$ Decay Chain Table 5: Relative Activity for Members of the a 08 Pu Decay Chain

Table 6: Relative Number of Atoms for Members of the ${ }^{2.9}$ Pu Decay Chain Table 7: Relative Activity for Members of the ${ }^{239} \mathrm{Pu}$ Decay Chain

Table 8: Relative Number of Atoms for Members of the $240 \mathrm{Pu}$ Decay Chain Table 9: Relative Activity for Members of the $24{ }^{\circ} \mathrm{Pu}$ Decay Chain

Table 10: Relative Number of Atoms for Members of the 241 Pu Decay Chain Table 11: Relative Activity for Members of the ${ }^{241} \mathrm{Pu}$ Decay Chain

Table 12: Relative Number of Atoms for Members of the a 4a Pu Decay Chain Table 13: Relative Activity for Members of the 24a Pu Decay Chain

Figure 7: Plot of the Relative Activities of the Members of the ${ }^{236} \mathrm{Pu}$ Decay Chain

Figure 8: Plot of the Relative Activities of the Members of the ${ }^{238} \mathrm{Pu}$ Decay Chain

Figure 9: Plot of the Relative Activities of the Members of the ${ }^{239} \mathrm{Pu}$ Decay Chain

Figure 10: Plot of the Relative Activities of the Members of the ${ }^{240} \mathrm{Pu}$ Decay Chain

Figure 11: Plot of the Relative Activities of the Members of the ${ }^{241} \mathrm{Pu}$ Decay Chain

Figure 12: Plot of the Relative Activities of the Members of the ${ }^{24^{2} \mathrm{Pu}}$ Decay Chain 


\section{Use of the Tables}

Two examples are given to show the use of these tables.

Example 1 - What is the intensity of the $2614 \mathrm{keV}$ gamma ray due to $208 \mathrm{~T} 1$ after ten years in a one kilogram plutonium metal heat source?

${ }^{308} \mathrm{~T} 1$ is a daughter of ${ }^{236} \mathrm{Pu}$, which is present in the source material in a concentration of $0.0001 \mathrm{wt} \%$; therefore, one milligram of ${ }^{236} \mathrm{Pu}$ is present in the one kilogram source. The original activity of ${ }^{2{ }^{6}} \mathrm{Pu}$ at the time the source was fabricated is given by

$$
\text { Activity }={ }^{\mathrm{N} \lambda} 236 \mathrm{Pu}
$$

where $\quad \mathrm{N}=$ number of atoms of ${ }^{3{ }^{3}} \mathrm{Pu}=\mathrm{N}_{\mathrm{O}} \mathrm{W} / \mathrm{M}$

$$
\begin{aligned}
& \mathrm{N}_{\circ}=\text { Avogadro's number }=6.023 \times 10^{33} \text { atoms } / \mathrm{mole} \\
& \mathrm{W}=\text { the weight of }{ }^{36} \mathrm{Pu} \text { in grams } \\
& \mathrm{M}=\text { the gram molecular weight } \\
& \lambda_{235 \mathrm{Pu}}=(\ln 2) /\left(\mathrm{T}^{\frac{1}{2}}\right)_{2{ }^{6}{ }^{6} \mathrm{Pu}} \\
& \mathrm{T}_{\frac{1}{2}}=\text { half-life of }{ }^{236} \mathrm{Pu}
\end{aligned}
$$

For the source under consideration, the activity is $1.967 \times 10^{10} \mathrm{dps}$. From Table 3 , at the end of ten years, the ${ }^{208} \mathrm{~T} 1$ activity is $1.067 \times 10^{-2}$ of the original ${ }^{236} \mathrm{Pu}$ activity or $2.099 \times 10^{8} \mathrm{dps}$. Since the $2614 \mathrm{keV}$ gamma ray is present [2] in $100 \%$ of the decays of ${ }^{208} \mathrm{Tl}$, the intensity of the $2614 \mathrm{keV}$ in the source is $2.099 \times 10^{8}$ gamma rays/ sec. The intensity of the $583 \mathrm{keV}$ transition, for instance, occurs in on $1 \mathrm{y} 86 \%$ of the decays [2] of ${ }^{208} \mathrm{Tl}$ and its intensity would be $1.805 \times 10^{8}$ gatnma rays $/ \mathrm{sec}$.

Example 2 - After eight years, what is the ratio of the intensities of the $59.6 \mathrm{keV}$ gamma ray due to the ingrowth of $241 \mathrm{Am}$ compared to the $43.5 \mathrm{keV}$ gamma ray in typical ${ }^{238} \mathrm{Pu}$ material ignoring self-absorption for both gamma rays in the source? 
The activity of ${ }^{3{ }^{3}} \mathrm{Pu}$ when the source was fabricated is given by

$$
\begin{aligned}
\text { Activity } & =\mathrm{N} \lambda_{2} 38 \mathrm{Pu} \\
& =\left(.8027 \mathrm{~W} \times \mathrm{N}_{0} / 238\right) \lambda_{238} \mathrm{Pu} \\
& =5.082 \times 10^{11} \mathrm{~W} \mathrm{dps}
\end{aligned}
$$

where $W=$ the weight in grams of the total plutonium present in the source.

The initial ${ }^{241} \mathrm{Pu}$ activity is

$$
\begin{aligned}
\text { Activity } & =\mathrm{N} \lambda_{241} \mathrm{Pu} \\
& =\left(.0072 \mathrm{~W} \times \mathrm{N}_{0} / 241\right) \lambda_{241} \mathrm{Pu} \\
& =2.702 \times 10^{10} \mathrm{Wdps}
\end{aligned}
$$

From Table 11, the relative activity of ${ }^{241} \mathrm{Am}$ after eight years is 0.01050 of the original activity of ${ }^{341} \mathrm{Pu}$. From Table 4 , the activity of ${ }^{3{ }^{3}} \mathrm{Pu}$ has decreased to 0.9388 of its original activity. From Lederer et a1. [2], the $59.6 \mathrm{keV}$ transition following the decay of ${ }^{341} \mathrm{Am}$ occurs in $36 \%$ of the decays of ${ }^{241 \mathrm{Am}}$; the $43.5 \mathrm{keV}$ transition following the decay of ${ }^{238} \mathrm{Pu}$ occurs $0.038 \%$ of the time. Therefore, after eight years the ratio of the intensity of the $43.5 \mathrm{keV}$ gamma ray from ${ }^{238} \mathrm{Pu}$ decay to the intensity of the $59.6 \mathrm{keV}$ gamma ray from $241 \mathrm{Am}$ decay is

$$
\begin{aligned}
& R=\frac{5.082 \times 10^{11} \times \mathrm{W} \times 3.8 \times 10^{-4} \times .9388}{2.702 \times 10^{10} \times \mathrm{W} \times .36 \times .01050} \\
& \mathrm{R}=1.78
\end{aligned}
$$

Similar examples can be worked for other daughter isotopes for other decay chains and for different time intervals. 
Table 2

RELATIVE NUMBER OF ATOMS FOR MEMBERS OF THE ${ }^{235} \mathrm{PU}$ DECAY CHAIN

\begin{tabular}{|c|c|c|c|c|c|c|c|c|c|c|c|}
\hline $\begin{array}{l}\text { time } \\
\text { (yr) }\end{array}$ & $\begin{array}{l}2{ }^{36} \mathrm{Pu} \\
(\times 1) \\
\end{array}$ & $\begin{array}{l}{ }^{2}{ }^{2} \mathrm{U} \\
(\times 1) \\
\end{array}$ & $\begin{array}{l}2 a \theta \mathrm{Th} \\
\left(\times 10^{-3}\right) \\
\end{array}$ & $\begin{array}{l}2 . \mathrm{Ra} \\
\left(\times 10^{-5}\right) \\
\end{array}$ & $\begin{array}{l}2{ }^{20} \mathrm{Rn} \\
\left(\times 10^{-3}\right) \\
\end{array}$ & $\begin{array}{l}2: 3 \mathrm{Po} \\
\left(\times 10^{-2}=2\right) \\
\end{array}$ & $\begin{array}{l}\therefore={ }^{2} \mathrm{~Pb} \\
\left(<10^{-\hat{3}}\right) \\
\end{array}$ & $\begin{array}{l}\text { a } 13 \mathrm{Bi} \\
\left(\times 10^{-7}\right) \\
\end{array}$ & $\begin{array}{l}308 \mathrm{Tl} \\
\left(\times 10^{-8}\right) \\
\end{array}$ & $\begin{array}{l}\text { a a } \mathrm{Po} \\
\left(\times 10^{-18}\right) \\
\end{array}$ & $\begin{array}{l}208 \mathrm{~Pb} \\
\left(\times 10^{-3}\right) \\
\end{array}$ \\
\hline 0.5 & 0.8855 & 0.1142 & 0.2643 & 0.1306 & 0.2296 & 0.6020 & 0.1579 & 0.1498 & 0.2759 & 0.8017 & .01514 \\
\hline 1 & 0.7841 & 0.2148 & 0.9561 & 0.4861 & 0.8547 & 2.241 & 0.5901 & 0.5600 & 1.031 & 2.995 & 0.1162 \\
\hline 2 & 0.6148 & 0.3812 & 3.140 & 1.620 & 2.848 & 7.467 & 1.970 & 1.870 & 3.443 & 10.00 & 0.8223 \\
\hline 4 & 0.3780 & 0.6083 & 8.613 & 4.474 & 7.866 & 20.63 & 5.446 & 5.169 & 9.519 & 27.66 & 5.034 \\
\hline 6 & 0.2324 & 0.7408 & 13.59 & 7.076 & 12.44 & 32.63 & 8.616 & 8.179 & 15.06 & 43.76 & 13.13 \\
\hline 8 & 0.1429 & 0.8153 & 17.34 & 9.037 & 15.89 & 41.66 & 11.00 & 10.45 & 19.24 & 55.89 & 24.41 \\
\hline 10 & 0.0879 & 0.8542 & 19.88 & 10.37 & 18.23 & 47.80 & 12.62 & 11.98 & 22.07 & 64.12 & 37.97 \\
\hline 12 & 0.0540 & 0.8714 & 21.47 & 11.20 & 19.69 & 51.62 & 13.64 & 12.94 & 23.84 & 69.25 & 53.02 \\
\hline 14 & 0.0332 & 0.8753 & 22.36 & 11.66 & 20.51 & 53.78 & 14.21 & 13.49 & 24.83 & 72.16 & 68.95 \\
\hline 16 & 0.0204 & 0.8713 & 22.78 & 11.89 & 20.90 & 54.80 & 14.48 & 13.74 & 25.30 & 73.53 & 85.35 \\
\hline 18 & 0.0126 & 0.8625 & 22.89 & 11.94 & 21.00 & 55.06 & 14.55 & 13.81 & 25.43 & 73.88 & 101.9 \\
\hline 20 & 0.0077 & 0.8508 & 22.80 & 11.90 & 20.92 & 54.85 & 14.49 & 13.75 & 25.33 & 73.59 & 118.5 \\
\hline 22 & 0.0047 & 0.8375 & 22.58 & 11.78 & 20.72 & 54.33 & 14.35 & 13.62 & 25.09 & 72.89 & 135.0 \\
\hline 24 & 0.0029 & 0.8234 & 22.29 & 11.63 & 20.45 & 53.62 & 14.16 & 13.45 & 24.76 & 71.94 & 151.3 \\
\hline 30 & 0.0007 & 0.7793 & 21.20 & 11.06 & 19.45 & 51.01 & 13.47 & 12.79 & 23.56 & 68.44 & 198.7 \\
\hline 40 & $6 \times 10^{-5}$ & 0.7084 & 19.30 & 10.07 & 17.71 & 46.44 & 12.27 & 11.64 & 21.44 & 62.31 & 272.2 \\
\hline 50 & $5 \times 10^{-5}$ & 0.6434 & 17.53 & 9.150 & 16.09 & 42.19 & 11.14 & 10.58 & 19.48 & 56.60 & 338.9 \\
\hline 60 & $5 \times 10^{-7}$ & 0.5844 & 15.92 & 8.310 & 14.61 & 38.31 & 10.12 & 9.608 & 17.69 & 51.41 & 399.6 \\
\hline 70 & $4 \times 10^{-3}$ & 0.5307 & 14.46 & 7.547 & 13.27 & 34.80 & 9.192 & 8.726 & 16.07 & 46.69 & 454.7 \\
\hline 90 & $3 \times 10^{-10}$ & 0.4378 & 11.93 & 6.226 & 10.75 & 28.70 & 7.583 & 7.198 & 13.26 & 38.51 & 550.2 \\
\hline 100 & $3 \times 10^{-11}$ & 0.3976 & 10.83 & 5.654 & $9.9+2$ & 26.07 & 6.887 & 6.537 & 12.04 & 34.98 & 591.5 \\
\hline 200 & $7.5 \times 10^{-22}$ & 0.1518 & 4.137 & 2.159 & 3.790 & 9.955 & 2.630 & 2.496 & 4.597 & 13.36 & 844.0 \\
\hline
\end{tabular}


Table 3

RELATIVE ACTIVITY FOR MEMBERS OF THE ${ }^{236} \mathrm{PU}$ DECAY CHAIN

\begin{tabular}{|c|c|c|c|c|c|c|c|c|c|c|}
\hline $\begin{array}{l}\text { Time } \\
(\mathrm{yr})\end{array}$ & $\begin{array}{l}{ }^{2{ }^{3}} \mathrm{Pu} \\
(\times 1) \\
\end{array}$ & $\begin{array}{l}{ }^{23} \mathrm{U} \\
\left(\times 10^{-2}\right) \\
\end{array}$ & $\begin{array}{l}\text { as } \mathrm{Th} \\
\left(\times 10^{-2}\right) \\
\end{array}$ & $\begin{array}{l}a^{24} \mathrm{Ra} \\
\left(\times 10^{-2}\right) \\
\end{array}$ & $\begin{array}{l}2{ }^{20} \mathrm{Rn} \\
\left(\times 10^{-a}\right) \\
\end{array}$ & $\begin{array}{l}226 \mathrm{Po} \\
\left(\times 10^{-2}\right) \\
\end{array}$ & $\begin{array}{l}218 \mathrm{~Pb} \\
\left(\times 10^{-2}\right) \\
\end{array}$ & $\begin{array}{l}213 \mathrm{Bi} \\
\left(\times 10^{-2}\right) \\
\end{array}$ & $\begin{array}{l}20 \mathrm{~T} 1 \\
\left(\times 10^{-2}\right) \\
\end{array}$ & $\begin{array}{l}213 \mathrm{Po} \\
\left(\times 10^{-2}\right) \\
\end{array}$ \\
\hline 0.5 & 0.8855 & 0.4520 & 0.03944 & 0.03735 & 0.03734 & 0.03734 & 0.03707 & 0.03705 & 0.01334 & 0.02372 \\
\hline 1 & 0.7841 & 0.8503 & 0.1427 & 0.1390 & 0.1390 & 0.1390 & 0.1386 & 0.1385 & 0.04985 & 0.08861 \\
\hline 2 & 0.6148 & 1.509 & 0.4685 & 0.4633 & 0.4632 & 0.4631 & 0.4626 & 0.4625 & 0.1665 & 0.2958 \\
\hline 4 & 0.3780 & 2.408 & 1.285 & 1.279 & 1.279 & 1.280 & 1.279 & 1.279 & 0.4603 & 0.8183 \\
\hline 6 & 0.2324 & 2.932 & 2.028 & 2.024 & 2.023 & 2.024 & 2.023 & 2.023 & 0.7282 & 1.295 \\
\hline 8 & 0.1429 & 3.227 & 2.587 & 2.584 & 2.584 & 2.584 & 2.583 & 2.585 & 0.9303 & 1.653 \\
\hline 10 & 0.0879 & 3.381 & 2.966 & 2.966 & 2.965 & 2.965 & 2.963 & 2.963 & 1.067 & 1.897 \\
\hline 12 & 0.0540 & 3.449 & 3.204 & 3.203 & 3.202 & 3.202 & 3.203 & 3.201 & 1.153 & 2.049 \\
\hline 14 & 0.0332 & 3.465 & 3.336 & 3.334 & 3.336 & 3.336 & 3.336 & 3.337 & 1.201 & 2.135 \\
\hline 16 & 0.0204 & 3.449 & 3.399 & 3.397 & 3.399 & 3.399 & 3.400 & 3.399 & 1.223 & 2.175 \\
\hline 18 & 0.0126 & 3.414 & 3.416 & 3.415 & 3.415 & 3.415 & 3.416 & 3.416 & 1.230 & 2.186 \\
\hline 20 & 0.0077 & 3.368 & 3.402 & 3.403 & 3.402 & 3.402 & 3.402 & 3.401 & 1.225 & 2.177 \\
\hline 22 & 0.0047 & 3.315 & 3.369 & 3.369 & 3.370 & 3.370 & 3.369 & 3.369 & 1.213 & 2.156 \\
\hline 24 & 0.0029 & 3.259 & 3.326 & 3.326 & 3.326 & 3.326 & 3.325 & 3.327 & 1.197 & 2.128 \\
\hline 30 & 0.0007 & 3.085 & 3.163 & 3.163 & 3.163 & 3.164 & 3.163 & 3.164 & 1.139 & 2.025 \\
\hline 40 & $6 \times 10^{-5}$ & 2.804 & 2.880 & 2.880 & 2.880 & 2.880 & 2.881 & 2.879 & 1.037 & 1.843 \\
\hline 50 & $5 \times 10^{-3}$ & 2.547 & 2.616 & 2.617 & 2.617 & 2.617 & 2.616 & 2.617 & 0.9419 & 1.674 \\
\hline 60 & $5 \times 10^{-7}$ & 2.313 & 2.375 & 2.376 & 2.376 & 2.376 & 2.376 & 2.377 & 0.8554 & 1.521 \\
\hline 70 & $4 \times 10^{-8}$ & 2.101 & 2.158 & 2.158 & 2.158 & 2.158 & 2.158 & 2.158 & 0.7770 & 1.381 \\
\hline 80 & $4 \times 10^{-5}$ & 1.908 & 1.961 & 1.960 & 1.960 & 1.960 & 1.960 & 1.960 & 0.7055 & 1.255 \\
\hline 90 & $3 \times 10^{-10}$ & 1.733 & 1.780 & 1.780 & 1.781 & 1.780 & 1.780 & 1.780 & 0.6412 & 1.139 \\
\hline 100 & $3 \times 10^{-11}$ & 1.574 & 1.616 & 1.617 & 1.617 & 1.616 & 1.617 & 1.617 & 0.5822 & 1.035 \\
\hline 200 & $7.5 \times 10^{-2 a}$ & 0.6009 & 0.6173 & 0.6174 & 0.6157 & 0.6175 & 0.6175 & 0.6174 & 0.2223 & 0.3953 \\
\hline
\end{tabular}


Table 4

RELATIVE NUMBER OF ATOMS FOR MEMBERS OF THE ${ }^{33} \mathrm{Pu}$ DECAY CHAIN

\begin{tabular}{|c|c|c|c|c|c|c|c|c|c|c|c|c|}
\hline $\begin{array}{l}\text { Time } \\
\text { (yr) } \\
\end{array}$ & $\begin{array}{l}{ }^{2{ }^{8}} \mathrm{Pu} \\
(\times 1) \\
\end{array}$ & $\begin{array}{l}234 \mathrm{U} \\
\left(\times 10^{-2}\right) \\
\end{array}$ & $\begin{array}{l}23^{\circ} \mathrm{Th} \\
\left(\times 10^{-7}\right) \\
\end{array}$ & $\begin{array}{l}2{ }^{28} \mathrm{Ra} \\
\left(\times 10^{-12}\right) \\
\end{array}$ & $\begin{array}{l}323 \mathrm{Rn} \\
\left(\times 10^{-17}\right) \\
\end{array}$ & $\begin{array}{l}310 \mathrm{Po} \\
\left(\times 10^{-21}\right) \\
\end{array}$ & $\begin{array}{l}\text { a } 14 \mathrm{~Pb} \\
\left(\times 10^{-18}\right) \\
\end{array}$ & $\begin{array}{l}21+\mathrm{Bi} \\
\left(\times 10^{-1 \theta}\right) \\
\end{array}$ & $\begin{array}{l}314 \mathrm{Po} \\
\left(\times 10^{-26}\right) \\
\end{array}$ & $\begin{array}{l}{ }^{1}{ }^{\circ} \mathrm{Pb} \\
\left(\times 10^{-13}\right)\end{array}$ & $\begin{array}{l}310 \mathrm{Bi} \\
\left(\times 10^{-13}\right) \\
\end{array}$ & $\begin{array}{l}310 \mathrm{Po} \\
\left(\times 10^{-25}\right)\end{array}$ \\
\hline 0.5 & 0.9961 & 0.3940 & 0.0276 & 0.043 & 0.0775 & & & & & & & \\
\hline 1 & 0.9921 & 0.7864 & 0.1105 & 0.064 & 0.0919 & & & & & & & \\
\hline 4 & 0.9689 & 3.108 & 1.754 & 1.869 & 1.255 & 6.337 & 0.6001 & 0.3480 & 0.4896 & 0.05268 & & \\
\hline 6 & 0.9537 & 4.626 & 3.926 & 7.492 & 4.910 & 26.50 & 2.394 & 1.660 & 2.317 & 0.1866 & 0.06135 & 0.1034 \\
\hline 8 & 0.9388 & 6.120 & 6.942 & 16.44 & 10.73 & 58.88 & 5.211 & 3.740 & 5.204 & 0.2390 & 0.09333 & 0.1673 \\
\hline 10 & 0.9241 & 7.591 & 10.79 & 31.56 & 20.57 & 113.4 & 10.01 & 7.276 & 10.09 & 0.4128 & 0.2065 & 0.4168 \\
\hline 12 & 0.9096 & 9.038 & 15.46 & 54.07 & 35.24 & 194.7 & 17.15 & 12.50 & 17.38 & 0.7225 & 0.4222 & 0.9333 \\
\hline 16 & 0.8813 & 11.87 & 27.20 & 127.1 & 82.85 & 458.2 & 40.32 & 29.54 & 41.00 & 2.080 & 1.327 & 3.167 \\
\hline 18 & 0.8675 & 13.25 & 34.25 & 179.8 & 117.2 & 648.8 & 57.05 & 41.83 & 58.07 & 3.188 & 2.067 & 5.042 \\
\hline 20 & 0.8539 & 14.61 & 42.06 & 245.4 & 160.0 & 886.0 & 77.90 & 57.17 & 79.35 & 4.724 & 3.104 & 7.673 \\
\hline 22 & 0.8406 & 15.94 & 506.4 & 325.3 & 212.2 & 1175 & 103.3 & 75.80 & 105.2 & 6.782 & 4.485 & 11.22 \\
\hline 24 & 0.8274 & 17.26 & 599.5 & 420.8 & 274.5 & 1520 & 133.6 & 98.13 & 136.2 & 9.476 & 6.288 & 15.88 \\
\hline 30 & 0.7891 & 21.09 & 92.26 & 812.7 & 530.2 & 2937 & 258.1 & 189.6 & 263.1 & 22.08 & 14.76 & 38.04 \\
\hline 40 & 0.7292 & 27.08 & 159.9 & 1886 & 1231 & 6821 & 599.4 & 440.5 & 611.2 & 64.41 & 43.19 & 113.4 \\
\hline 50 & 0.6739 & 32.61 & 243.8 & 3613 & 2359 & 13070 & 1148 & 844.0 & 1171 & 146.3 & 98.23 & 260.6 \\
\hline 60 & 0.6227 & 37.73 & 342.5 & 6122 & 3997 & 22150 & 1946 & 1430 & 1985 & 282.7 & 189.9 & 507.5 \\
\hline 70 & 0.5754 & 42.45 & 455.1 & 9535 & 6226 & 34490 & 3031 & 2228 & 3091 & 488.8 & 328.5 & 882.3 \\
\hline 90 & 0.4914 & 50.85 & 717.4 & 19500 & 12740 & 70570 & 6201 & 4558 & 6324 & 1171 & 787.2 & 2129 \\
\hline 100 & 0.4541 & 54.58 & 865.4 & 26250 & 17150 & 95000 & 8347 & 6136 & 8513 & 1676 & 1127 & 3054 \\
\hline 200 & 0.2062 & 79.35 & 2788 & 175800 & 114800 & 636200 & 55910 & 41090 & 57020 & 15490 & 10420 & 28520 \\
\hline
\end{tabular}


Table 5

RELATIVE ACTIVITY FOR MEMBERS OF THE ${ }^{30}$ Pu DECAY CHAIN

\begin{tabular}{|c|c|c|c|c|c|c|c|c|c|c|c|c|}
\hline $\begin{array}{l}\text { Time } \\
(\mathrm{yr}) \\
\end{array}$ & $\begin{array}{l}23^{8} \mathrm{Pu} \\
(\times 1) \\
\end{array}$ & $\begin{array}{l}234 \mathrm{U} \\
\left(\times 10^{-8}\right) \\
\end{array}$ & $\begin{array}{l}330 \mathrm{Th} \\
\left(\times 10^{-11}\right) \\
\end{array}$ & $\begin{array}{l}228 \mathrm{Ra} \\
\left(\times 10^{-1 a}\right) \\
\end{array}$ & $\begin{array}{l}2{ }^{2} \mathrm{Rn} \\
\left(\times 10^{-1 a}\right) \\
\end{array}$ & $\begin{array}{l}\text { a } 18 \mathrm{Po} \\
\left(\times 10^{-13}\right) \\
\end{array}$ & $\begin{array}{l}214 \mathrm{~Pb} \\
\left(\times 10^{-12}\right) \\
\end{array}$ & $\begin{array}{l}\text { a } 4 \mathrm{Bi} \\
\left(\times 10^{-12}\right) \\
\end{array}$ & $\begin{array}{l}314 \mathrm{Po} \\
\left(\times 10^{-13}\right) \\
\end{array}$ & $\begin{array}{l}210 \mathrm{~Pb} \\
\left(\times 10^{-12}\right) \\
\end{array}$ & $\begin{array}{l}210 \mathrm{Bi} \\
\left(\times 10^{-12}\right) \\
\end{array}$ & $\begin{array}{l}210 \mathrm{Po} \\
\left(\times 10^{-13}\right)\end{array}$ \\
\hline 0.5 & 0.9961 & 1.401 & 0.3029 & 0.0024 & 0.0065 & & & & & & & \\
\hline 1 & 0.9921 & 2.795 & 1.213 & 0.0035 & 0.0077 & & & & & & & \\
\hline 2 & 0.9843 & 5.570 & 4.838 & 0.0197 & 0.0232 & 0.0131 & 0.0210 & 0.00056 & 0.0027 & & & \\
\hline 4 & 0.9689 & 11.05 & 19.25 & 0.1024 & 0.1053 & 0.0959 & 0.1034 & 0.0816 & 0.0827 & 0.0227 & & \\
\hline 6 & 0.9537 & 16.44 & 43.09 & 0.4106 & 0.4119 & 0.4012 & 0.4125 & 0.3891 & 0.3914 & 0.0803 & 0.03925 & 0.02396 \\
\hline 8 & 0.9388 & 21.75 & 76.19 & 0.9010 & 0.9001 & 0.8915 & 0.8979 & 0.8767 & 0.8792 & 0.1029 & 0.05970 & 0.03876 \\
\hline 10 & 0.9241 & 26.98 & 118.4 & 1.730 & 1.726 & 1.717 & 1.725 & 1.705 & 1.705 & 0.1777 & 0.1321 & 0.09658 \\
\hline 12 & 0.9096 & 32.13 & 169.7 & 2.963 & 2.956 & 2.948 & 2.955 & 2.930 & 2.936 & 0.3110 & 0.2701 & 0.2163 \\
\hline 14 & 0.8954 & 37.18 & 229.7 & 4.680 & 4.669 & 4.660 & 4.664 & 4.644 & 4.648 & 0.5401 & 0.4960 & 0.4148 \\
\hline 16 & 0.8813 & 42.19 & 298.5 & 6.966 & 6.950 & 6.937 & 6.947 & 6.924 & 6.927 & 0.8952 & 0.8489 & 0.7338 \\
\hline 18 & 0.8675 & 47.10 & 375.9 & 9.854 & 9.831 & 9.823 & 9.830 & 9.805 & 9.811 & 1.372 & 1.322 & 1.168 \\
\hline 20 & 0.8539 & 51.93 & 461.6 & 13.45 & 13.42 & 13.41 & 13.42 & 13.40 & 13.41 & 2.033 & 1.986 & 1.778 \\
\hline 22 & 0.8406 & 56.66 & 555.8 & 17.83 & 17.80 & 17.79 & 17.80 & 17.77 & 17.77 & 2.919 & 2.869 & 2.600 \\
\hline 24 & 0.8274 & 61.35 & 658.0 & 23.06 & 23.11 & 23.01 & 23.02 & 23.00 & 23.01 & 4.078 & 4.022 & 3.680 \\
\hline 30 & 0.7891 & 74.97 & 1013 & 44.54 & 44.48 & 44.47 & 44.47 & 44.44 & 44.45 & 9.503 & 9.442 & 8.814 \\
\hline 40 & 0.7292 & 96.26 & 1755 & 103.4 & 103.3 & 103.3 & 103.3 & 103.3 & 103.3 & 27.72 & 27.63 & 26.28 \\
\hline 50 & 0.6739 & 115.9 & 2676 & 198.0 & 197.9 & 197.9 & 197.8 & 197.8 & 197.8 & 62.97 & 62.84 & 60.38 \\
\hline 60 & 0.6227 & 134.1 & 3759 & 335.5 & 335.3 & 335.4 & 335.3 & 335.2 & 335.4 & 121.7 & 121.5 & 117.6 \\
\hline 70 & 0.5754 & 150.9 & 4995 & 522.6 & 522.3 & 522.2 & 522.3 & 522.3 & 522.2 & 210.4 & 210.1 & 204.4 \\
\hline 80 & 0.5318 & 166.4 & 6370 & 765.1 & 764.8 & 764.7 & 764.7 & 764.6 & 764.8 & 335.7 & 335.4 & 327.6 \\
\hline 90 & 0.4914 & 180.8 & 7873 & 1069 & 1069 & 1068 & 1068 & 1068 & 1068 & 504.0 & 503.6 & 493.3 \\
\hline 100 & 0.4541 & 194.0 & 9498 & 1439 & 1439 & 1438 & 1438 & 1438 & 1438 & 721.3 & 720.9 & 707.6 \\
\hline 200 & 0.2062 & 282.1 & 30600 & 9635 & 9630 & 9632 & 9634 & 9632 & 9633 & 6667 & 6666 & 6608 \\
\hline
\end{tabular}


Table 6

RELATIVE NUMBER OF ATOMS FOR MEMBERS OF THE ${ }^{3{ }^{3 y}}$ Pu DECAY CHAIN

\begin{tabular}{|c|c|c|c|c|c|c|c|c|c|c|c|c|}
\hline $\begin{array}{l}\text { Time } \\
(\mathrm{yr})\end{array}$ & $\begin{array}{c}2{ }^{29} \mathrm{Pu} \\
(\times 1) \\
\end{array}$ & $\begin{array}{l}235 \mathrm{U} \\
\left(\times 10^{-4}\right) \\
\end{array}$ & $\begin{array}{l}831 \mathrm{Th} \\
\left(\times 10^{-15}\right) \\
\end{array}$ & $\begin{array}{l}\text { a s } \mathrm{Pa} \\
\left(\times 10^{-13}\right) \\
\end{array}$ & $\begin{array}{l}337 \mathrm{AC} \\
\left(\times 10^{-1 \mathrm{E}}\right) \\
\end{array}$ & $\begin{array}{l}\text { a }{ }^{7} \mathrm{Th} \\
\left(\times 10^{-17}\right) \\
\end{array}$ & $\begin{array}{l}{ }^{a}{ }^{3} \mathrm{Ra} \\
\left(\times 10^{-1{ }^{8}}\right) \\
\end{array}$ & $\begin{array}{l}\text { a } 19 \mathrm{Rn} \\
\left(\times 10^{-23}\right)\end{array}$ & $\begin{array}{l}315 \mathrm{Po} \\
\left(\times 10^{-37}\right) \\
\end{array}$ & $\begin{array}{l}211 \mathrm{~Pb} \\
\left(\times 10^{-a \circ}\right) \\
\end{array}$ & $\begin{array}{l}811 \mathrm{Bi} \\
\left(\times 10^{-31}\right) \\
\end{array}$ & $\begin{array}{l}207 \mathrm{~T} 1 \\
\left(\times 10^{-31}\right) \\
\end{array}$ \\
\hline 0.5 & .9999 & .1421 & .0578 & & & & & & & & & \\
\hline 1 & .9999 & .2842 & .1160 & .007 & & & & & & & & \\
\hline 2 & .9999 & .5684 & .2326 & .041 & & & & & & & & \\
\hline 4 & .9999 & 1.137 & .4656 & .217 & .0051 & & & .0033 & .0143 & .0032 & .0016 & .0033 \\
\hline 6 & .9998 & 1.705 & .6986 & .483 & .0118 & .0013 & .0042 & .0064 & .0300 & .0052 & .0028 & .0055 \\
\hline 8 & .9998 & 2.273 & .9316 & .886 & .0495 & .0100 & .0535 & .0281 & .1232 & .0169 & .0098 & .0210 \\
\hline 10 & .9997 & 2.841 & 1.165 & 1.376 & .0861 & .0183 & .1055 & .0495 & .2206 & .0283 & .0165 & .0365 \\
\hline 12 & .9997 & 3.410 & 1.398 & 1.996 & .1570 & .0344 & .2054 & .0898 & .3976 & .0501 & .0297 & .0655 \\
\hline 14 & .9996 & 3.978 & 1.631 & 2.710 & .2407 & .0532 & .3242 & .1377 & .6109 & .0761 & .0452 & .0998 \\
\hline 16 & .9995 & 4.546 & 1.864 & 3.534 & .3492 & .0782 & .4793 & .2010 & .8938 & .1104 & .0657 & .1454 \\
\hline 18 & .9995 & 5.114 & 2.096 & 4.481 & .4954 & .1119 & .6901 & .2861 & 1.271 & .1565 & .0932 & .2065 \\
\hline 20 & .9994 & 5.682 & 2.329 & 5.524 & .6652 & .1510 & .9326 & .3842 & 1.707 & .2096 & .1249 & .2768 \\
\hline 22 & .9994 & 6.250 & 2.562 & 6.699 & .8831 & .2006 & 1.244 & .5106 & 2.268 & .2780 & .1659 & .3678 \\
\hline 24 & .9993 & 6.818 & 2.795 & 7.983 & 1.137 & .2588 & 1.609 & .6583 & 2.926 & .3581 & .2139 & .4738 \\
\hline 30 & .9992 & 8.522 & 3.494 & 12.46 & 2.121 & .4847 & 3.026 & 1.231 & 5.473 & .6684 & .3996 & .8857 \\
\hline 40 & .9989 & 11.36 & 4.658 & 22.17 & 4.708 & 1.080 & 6.754 & 2.742 & 12.19 & 1.486 & .8887 & 1.971 \\
\hline 50 & .9986 & 14.20 & 5.822 & 34.65 & 8.624 & 1.981 & 12.41 & 5.030 & 22.36 & 2.725 & 1.630 & 3.615 \\
\hline 60 & .9983 & 17.04 & 6.985 & 49.87 & 13.99 & 3.215 & 20.15 & 8.166 & 36.30 & 4.423 & 2.646 & 5.868 \\
\hline 70 & .9980 & 19.87 & 8.149 & 67.88 & 20.94 & 4.817 & 30.21 & 12.24 & 54.39 & 6.627 & 3.965 & 8.792 \\
\hline 80 & .9977 & 22.71 & 9.311 & 88.66 & 29.53 & 6.796 & 42.63 & 17.27 & 76.75 & 9.352 & 5.595 & 12.41 \\
\hline 90 & .9974 & 25.54 & 10.47 & 112.2 & 39.81 & 9.165 & 57.50 & 23.29 & 103.5 & 12.61 & 7.545 & 16.73 \\
\hline 100 & .9972 & 28.38 & 11.64 & 138.5 & 51.85 & 11.94 & 74.90 & 30.33 & 134.8 & 16.43 & 9.828 & 21.79 \\
\hline 200 & .9943 & 56.68 & 23.24 & 553.0 & 270.9 & 62.45 & 392.2 & 158.8 & 705.8 & 85.98 & 51.44 & 114.1 \\
\hline 400 & .9887 & 113.0 & 46.35 & 2205 & 1255 & 289.5 & 1818 & 736.2 & 3273 & 398.7 & 238.5 & 529.0 \\
\hline 600 & .9831 & 169.1 & 69.33 & 4945 & 2964 & 683.6 & 4294 & 1738 & 7728 & 941.5 & 563.3 & 1249 \\
\hline 800 & .9775 & 224.8 & 92.17 & 8761 & 5390 & 1243 & 7809 & 3162 & 14050 & 1712 & 1024 & 2272 \\
\hline 1000 & .9720 & 280.2 & 114.9 & 13640 & 8525 & 1966 & 12350 & 5001 & 22230 & 2708 & 1620 & 3594 \\
\hline
\end{tabular}


Table 7

RELATIVE ACTIVITY FOR MEMBERS OF THE ${ }^{23}{ }^{9} \mathrm{Pu}$ DECAY CHAIN

\begin{tabular}{|c|c|c|c|c|c|c|c|c|c|c|c|c|}
\hline $\begin{array}{l}\text { Time } \\
(\mathrm{yr}) \\
\end{array}$ & $\begin{array}{l}3{ }^{3}{ }^{9} \mathrm{Pu} \\
(\times 1) \\
\end{array}$ & $\begin{array}{l}{ }^{3{ }^{5}} \mathrm{U} \\
\left(\times 10^{-8}\right) \\
\end{array}$ & $\begin{array}{l}231 \mathrm{Th} \\
\left(\times 10^{-8}\right) \\
\end{array}$ & $\begin{array}{l}232 \mathrm{~Pa} \\
\left(\times 10^{-13}\right) \\
\end{array}$ & $\begin{array}{l}a^{27} \mathrm{AC} \\
\left(\times 10^{-12}\right)\end{array}$ & $\begin{array}{l}2^{7} \mathrm{Th} \\
\left(\times 10^{-12}\right) \\
\end{array}$ & $\begin{array}{l}2^{2}{ }^{3} \mathrm{Ra} \\
\left(\times 10^{-12}\right) \\
\end{array}$ & $\begin{array}{l}2{ }^{18} \mathrm{Rn} \\
\left(\times 10^{-1 a}\right) \\
\end{array}$ & $\begin{array}{l}{ }^{15} \mathrm{Po} \\
\left(\times 10^{-1 \bar{\varepsilon}}\right)\end{array}$ & $\begin{array}{l}311 \mathrm{~Pb} \\
\left(\times 10^{-12}\right) \\
\end{array}$ & $\begin{array}{l}{ }^{112} \mathrm{Bi} \\
\left(\times 10^{-12}\right) \\
\end{array}$ & $\begin{array}{l}207 \mathrm{~T} 1 \\
\left(\times 10^{-12}\right) \\
\end{array}$ \\
\hline 0.5 & .9999 & .0488 & .0484 & & & & & & & & & \\
\hline 1 & .9999 & .0976 & .0972 & .0052 & & & & & & & & \\
\hline 2 & .9999 & .1953 & .1949 & .0308 & & & & & & & & \\
\hline 4 & .9999 & .3906 & .3900 & .1629 & .0058 & & & .0063 & .0062 & .0115 & .0094 & .0089 \\
\hline 6 & .9998 & .5857 & .5853 & .3625 & .0133 & .0066 & .0003 & .0124 & .0130 & .0186 & .0169 & .0147 \\
\hline 8 & .9998 & .7808 & .7805 & .6649 & .0560 & .0490 & .0417 & .0541 & .0533 & .0601 & .0581 & .0563 \\
\hline 10 & .9997 & .9763 & .9752 & 1.033 & .0972 & .0894 & .0822 & .0952 & .0955 & .1006 & .0980 & .0977 \\
\hline 12 & .9997 & 1.171 & 1.171 & 1.498 & .1773 & .1684 & .1601 & .1728 & .1721 & .1786 & 1763 & .1753 \\
\hline 14 & .9996 & 1.367 & 1.367 & 2.034 & .2718 & .2605 & .2526 & .2650 & .2645 & .2705 & .2682 & .2674 \\
\hline 16 & .9995 & 1.562 & 1.561 & 2.652 & .3943 & .3830 & .3736 & .3868 & .3869 & .3922 & .3899 & .3893 \\
\hline 18 & .9995 & 1.757 & 1.756 & 3.363 & .5594 & .5477 & .5378 & .5505 & .5502 & .5561 & .5535 & .5529 \\
\hline 20 & .9994 & 1.952 & 1.951 & 4.146 & .7511 & .7421 & .7268 & .7393 & .7389 & .7448 & .7419 & .7413 \\
\hline 22 & .9994 & 2.147 & 2.146 & 5.027 & .9972 & .9819 & .9695 & .9825 & .9818 & .9879 & .9855 & .9850 \\
\hline 24 & .9993 & 2.342 & 2.342 & 5.991 & 1.284 & 1.267 & 1.254 & 1.267 & 1.267 & 1.272 & 1.270 & 1.269 \\
\hline 30 & .9992 & 2.927 & 2.927 & 9.351 & 2.395 & 2.373 & 2.358 & 2.369 & 2.370 & 2.375 & 2.373 & 2.372 \\
\hline 40 & .9989 & 3.902 & 3.902 & 16.64 & 5.315 & 5.286 & 5.264 & 5.274 & 5.277 & 5.281 & 5.278 & 5.278 \\
\hline 50 & .9986 & 4.878 & 4.877 & 26.00 & 9.738 & 9.696 & 9.683 & 9.679 & 9.679 & 9.684 & 9.682 & 9.680 \\
\hline 60 & .9983 & 5.854 & 5.852 & 37.43 & 15.80 & 15.74 & 15.70 & 15.71 & 15.71 & 15.72 & 15.72 & 15.72 \\
\hline 70 & .9980 & 6.826 & 6.827 & 50.94 & 23.64 & 23.58 & 23.54 & 23.55 & 23.54 & 23.55 & 23.55 & 23.55 \\
\hline 80 & .9977 & 7.801 & 7.800 & 66.54 & 33.37 & 33.26 & 33.22 & 33.23 & 33.22 & 33.23 & 33.23 & 33.23 \\
\hline 90 & .9974 & 8.774 & 8.771 & 84.20 & 44.95 & 44.85 & 44.81 & 44.81 & 44.80 & 44.81 & 44.81 & 44.81 \\
\hline 100 & .9972 & 9.749 & 9.752 & 103.9 & 58.52 & 58.44 & 58.38 & 58.36 & 58.35 & 58.37 & 58.36 & 58.36 \\
\hline 200 & .9943 & 19.47 & 19.47 & 415.0 & 305.9 & 305.7 & 305.7 & 305.4 & 305.5 & 305.5 & 305.5 & 305.5 \\
\hline 400 & .9887 & 38.82 & 38.83 & 1654 & 1417 & 1417 & 1417 & $141 ?$ & 1417 & 1417 & 1417 & 1417 \\
\hline 600 & .9831 & 58.09 & 58.08 & 3711 & 3347 & 3346 & 3347 & 3344 & 3345 & 3345 & 3345 & 3345 \\
\hline 800 & .9775 & 77.22 & 77.22 & 6575 & 6086 & 6084 & 6086 & 6084 & 6082 & 6084 & 6084 & 6084 \\
\hline 1000 & .9720 & 96.25 & 96.25 & 10240 & 9626 & 9623 & 9625 & 9623 & 9623 & 9624 & 9624 & 9624 \\
\hline
\end{tabular}


Table 8

RELATIVE NUMBER OF ATOMS FOR MEMBERS OF THE $24{ }^{\circ} \mathrm{Pu}$ DECAY CHAIN

\begin{tabular}{|c|c|c|c|c|c|c|}
\hline $\begin{array}{l}\text { Time } \\
(\mathrm{yr}) \\
\end{array}$ & $\begin{array}{l}24^{\circ} \mathrm{Pu} \\
(\times 1) \\
\end{array}$ & $\begin{array}{l}\begin{array}{l}3 \\
\left(\times 10^{-3}\right) \\
\end{array} \\
\end{array}$ & $\begin{array}{l}2{ }^{2} \mathrm{Th} \\
\left(\times 10^{-8}\right) \\
\end{array}$ & $\begin{array}{l}2^{28} \mathrm{Ra} \\
\left(\times 10^{-18}\right) \\
\end{array}$ & $\begin{array}{l}{ }^{2}{ }^{\theta} \mathrm{Ac} \\
\left(\times 10^{-22}\right)\end{array}$ & $\begin{array}{l}z 28 \mathrm{Th} \\
\left(\because 10^{-18}\right)\end{array}$ \\
\hline 0.5 & .9999 & .0537 & & & & \\
\hline 1 & .9999 & .1053 & & .0386 & .0482 & \\
\hline 2 & .9998 & .2107 & .01 & .0426 & .0518 & \\
\hline 4 & .9996 & .4213 & .03 & .0442 & .0543 & \\
\hline 6 & .9994 & .6318 & .05 & .0490 & .0568 & \\
\hline 8 & .9992 & .8424 & .09 & .0537 & .0631 & \\
\hline 10 & .9990 & 1.053 & .15 & .0637 & .0746 & \\
\hline 12 & .9987 & 1.263 & .19 & .0678 & .0780 & 0.0194 \\
\hline 14 & .9985 & 1.474 & .28 & .0901 & .1006 & 0.0243 \\
\hline 16 & .9983 & 1.684 & .37 & .1071 & .1189 & 0.0277 \\
\hline 18 & .9981 & 1.894 & .49 & .1419 & .1550 & 0.0356 \\
\hline 20 & .9979 & 2.105 & .61 & .1738 & .1881 & 0.0432 \\
\hline 22 & .9977 & 2.315 & .72 & .2061 & .2214 & 0.0504 \\
\hline 24 & .9975 & 2.525 & .89 & .2557 & .2742 & 0.0635 \\
\hline 30 & .9968 & 3.155 & 1.39 & .4119 & .4376 & 0.1026 \\
\hline 40 & .9958 & 4.205 & 2.44 & .7748 & .8157 & 0.1941 \\
\hline 50 & .9948 & 5.253 & 3.81 & 1.289 & 1.352 & 0.3297 \\
\hline 60 & .9937 & 6.301 & 5.48 & 1.943 & 2.035 & 0.5039 \\
\hline 70 & .9927 & 7.347 & 7.46 & 2.748 & 2.875 & 0.7230 \\
\hline 80 & .9916 & 8.392 & 9.75 & 3.695 & 3.864 & 0.9820 \\
\hline 90 & .9906 & 9.436 & 12.34 & 4.788 & 5.005 & 1.282 \\
\hline 100 & .9895 & 10.48 & 15.22 & 6.014 & 6.285 & 1.620 \\
\hline 200 & .9792 & 20.85 & 60.65 & 26.22 & 27.38 & 7.267 \\
\hline 400 & .9587 & 41.26 & 241.0 & 109.2 & 113.9 & 30.70 \\
\hline 600 & .9387 & 61.25 & 538.5 & 247.9 & 258.7 & 70.02 \\
\hline 800 & .9192 & 80.82 & 950.7 & 441.2 & 460.5 & 124.9 \\
\hline 1000 & .9000 & 99.98 & 1475 & 687.9 & 718.0 & 195.0 \\
\hline
\end{tabular}


Table 9

RELATIVE ACTIVITY FOR MEMBERS OF THE ${ }^{24}{ }^{\circ} \mathrm{Pu}$ DECAY CHAIN

\begin{tabular}{|c|c|c|c|c|c|c|}
\hline $\begin{array}{l}\text { Time } \\
\text { (yr) } \\
\end{array}$ & $\begin{array}{l}24^{\circ} \mathrm{Pu} \\
(\times 1) \\
\end{array}$ & $\begin{array}{l}2{ }^{36} \mathrm{U} \\
\left(\times 10^{-6}\right) \\
\end{array}$ & $\begin{array}{l}2 \approx \bar{c}_{\mathrm{Th}} \\
\left(\times 10^{-1}=\right) \\
\end{array}$ & $\begin{array}{l}2{ }^{28} \mathrm{Ra} \\
\left(\times 10^{-15}\right) \\
\end{array}$ & $\begin{array}{l}2{ }^{8} \mathrm{Ac} \\
\left(\times 10^{-15}\right) \\
\end{array}$ & $\begin{array}{l}23{ }^{8} \mathrm{Th} \\
\left(\times 10^{-15}\right) \\
\end{array}$ \\
\hline 0.5 & .9999 & .0145 & & & & \\
\hline 1 & .9999 & .0290 & & .0379 & .0454 & \\
\hline 2 & .9998 & .0580 & .0047 & .0418 & .0487 & \\
\hline 4 & .9996 & .1160 & .0140 & .0434 & .0511 & \\
\hline 6 & .9994 & .1740 & .0233 & .0481 & .0535 & \\
\hline 8 & .9992 & .2319 & .0420 & .0527 & .0593 & \\
\hline 10 & .9990 & .2899 & .0700 & .0626 & .0702 & \\
\hline 12 & .9987 & .3478 & .0887 & .0665 & .0734 & .0667 \\
\hline 14 & .9985 & .4057 & .1307 & .0885 & .0948 & .0837 \\
\hline 16 & .9983 & .4636 & .1727 & .1051 & .1119 & .0953 \\
\hline 18 & .9981 & .5215 & .2287 & .1394 & .1458 & .1226 \\
\hline 20 & .9979 & .5794 & .2847 & .1707 & .1770 & .1487 \\
\hline 22 & .9977 & .6373 & .3360 & .2024 & .2084 & .1738 \\
\hline 24 & .9975 & .6952 & .4153 & .2510 & .2580 & .2187 \\
\hline 30 & .9968 & .8687 & .6487 & .4045 & .4118 & .3534 \\
\hline 40 & .9958 & 1.158 & 1.139 & .7610 & .7676 & .6688 \\
\hline 50 & .9948 & 1.446 & 1.778 & 1.266 & 1.272 & 1.136 \\
\hline 60 & .9937 & 1.735 & 2.557 & 1.908 & 1.915 & 1.736 \\
\hline 70 & .9927 & 2.023 & 3.481 & 2.670 & 2.705 & 2.491 \\
\hline 80 & .9916 & 2.310 & 4.550 & 3.629 & 3.636 & 3.383 \\
\hline 90 & .9906 & 2.598 & 5.759 & 4.702 & 4.709 & 4.417 \\
\hline 100 & .9895 & 2.885 & 7.103 & 5.906 & 5.914 & 5.581 \\
\hline 200 & .9792 & 5.740 & 28.30 & 25.75 & 25.75 & 25.04 \\
\hline 400 & .9587 & 11.36 & 112.5 & 107.2 & 107.2 & 105.8 \\
\hline 600 & .9387 & 16.86 & 251.3 & 243.4 & 243.5 & 241.2 \\
\hline 800 & .9192 & 22.25 & 443.7 & 433.3 & 433.3 & 430.3 \\
\hline 1000 & .9000 & 27.53 & 688.5 & 675.5 & 675.6 & 671.9 \\
\hline
\end{tabular}


Table 10

RELATIVE NUMBER OF ATOMS FOR MEMBERS OF THE $\approx 41 \mathrm{PU}$ DECAY CHAIN

\begin{tabular}{|c|c|c|c|c|c|c|c|}
\hline $\begin{array}{l}\text { Time } \\
(\mathrm{yr}) \\
\end{array}$ & $\begin{array}{l}24 \perp \mathrm{Pu} \\
(\times 1) \\
\end{array}$ & $\begin{array}{l}24 \mathrm{Am} \\
(\times 1) \\
\end{array}$ & $\begin{array}{l}3:{ }^{7} \mathrm{~Np} \\
\left(\times 10^{-4}\right) \\
\end{array}$ & $\begin{array}{l}253 \mathrm{~Pa} \\
\left(\times 10^{-11}\right) \\
\end{array}$ & $\begin{array}{l}2: 3{ }^{3} U \\
\left(\times 10^{-5}\right) \\
\end{array}$ & $\begin{array}{l}2:=\mathrm{Th} \\
\left(\times 10^{-11}\right) \\
\end{array}$ & $\begin{array}{l}225 \mathrm{Ra} \\
\left(\times 10^{-2}=1\right) \\
\end{array}$ \\
\hline 0.5 & 0.9762 & 0.02382 & 0.09528 & 0.02186 & & & \\
\hline 1 & 0.9529 & 0.04705 & 0.3780 & 0.1058 & 0.00038 & & \\
\hline 2 & 0.9080 & 0.09182 & 1.487 & 0.4626 & 0.00275 & & \\
\hline 4 & 0.8245 & 0.1749 & 5.759 & 1.889 & 0.02336 & & \\
\hline 6 & 0.7487 & 0.2500 & 12.55 & 4.191 & 0.07900 & & \\
\hline 8 & 0.6798 & 0.3180 & 21.62 & 7.284 & 0.1854 & & \\
\hline 10 & 0.6173 & 0.3794 & 32.75 & 11.09 & 0.3566 & & \\
\hline 12 & 0.5606 & 0.4349 & 45.74 & 15.55 & 0.6058 & & \\
\hline 14 & 0.5090 & 0.4850 & 60.41 & 20.58 & 0.9438 & & \\
\hline 16 & 0.4622 & 0.5301 & 76.60 & 26.15 & 1.381 & 0.0214 & 0.01 \\
\hline 18 & 0.4197 & 0.5709 & 94.15 & 32.19 & 1.927 & 0.0340 & 0.0500 \\
\hline 20 & 0.3811 & 0.6076 & 112.9 & 38.66 & 2.591 & 0.0513 & 0.1671 \\
\hline 22 & 0.3460 & 0.6407 & 132.8 & 45.51 & 3.380 & 0.0745 & 0.2709 \\
\hline 24 & 0.3142 & 0.6704 & 153.7 & 52.72 & 4.300 & 0.1053 & 0.4348 \\
\hline 30 & 0.2353 & 0.7426 & 221.5 & 76.07 & 7.911 & 0.2558 & 1.272 \\
\hline 40 & 0.1452 & 0.8201 & 346.6 & 119.2 & 17.03 & 0.7782 & 4.140 \\
\hline 50 & $8.965 \times 10^{-2}$ & 0.8622 & 481.0 & 165.6 & 30.37 & 1.767 & 9.570 \\
\hline 60 & $5.535 \times 10^{--}$ & 0.8826 & 620.2 & 213.7 & 48.17 & 3.454 & 18.89 \\
\hline 70 & $3.417 \times 10^{-2}$ & 0.8897 & 761.5 & 262.5 & 70.47 & 5.958 & 32.65 \\
\hline 80 & $2.109 \times 10^{-2}$ & 0.8886 & 903.3 & 311.5 & 97.38 & 9.527 & 52.32 \\
\hline 90 & $1.302 \times 10^{-2}$ & 0.8825 & 1044 & 360.3 & 128.9 & 14.32 & 78.76 \\
\hline 100 & $8.038 \times 10^{-3}$ & 0.8735 & 1184 & 408.6 & 164.9 & 20.56 & 113.2 \\
\hline 200 & $6.461 \times 10^{-5}$ & 0.7519 & 2481 & 856.4 & 763.1 & 203.3 & 1121 \\
\hline 400 & $4.174 \times 10^{-}=$ & 0.5467 & 4532 & 1565 & 3068 & 1733 & 9561 \\
\hline 600 & $2.697 \times 10^{-13}$ & 0.3975 & 6024 & 2081 & 6508 & 5695 & 31430 \\
\hline 800 & $1.743 \times 10^{-17}$ & 0.2890 & 7108 & 2455 & 10770 & 12870 & 71030 \\
\hline 1000 & $1.126 \times 10^{-21}$ & 0.2102 & 7897 & 2728 & 15630 & 23790 & 131300 \\
\hline
\end{tabular}


Table 10 (continued)

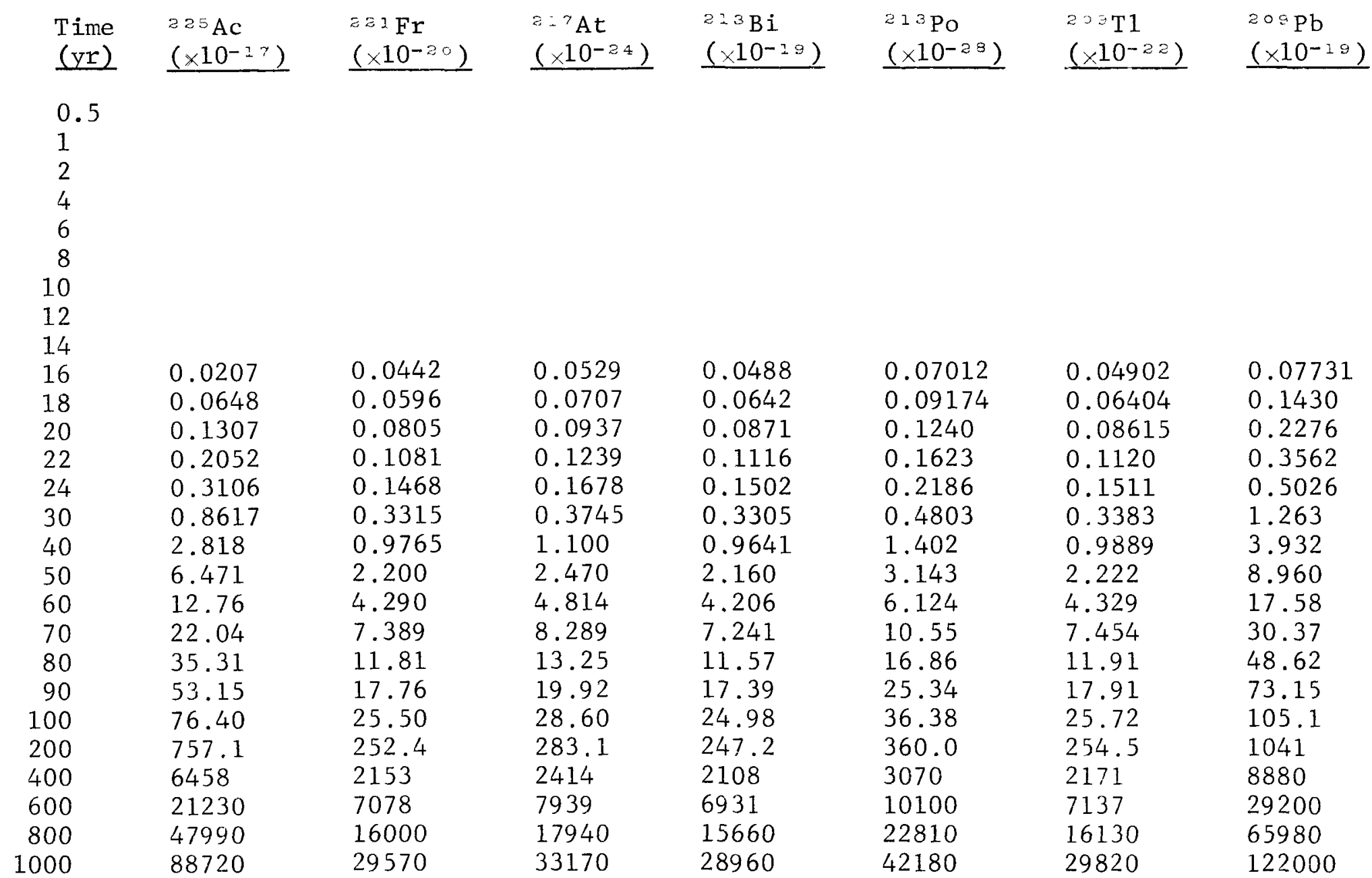


Table 11

RELATIVE ACTIVITY FOR MEMBERS OF THE ${ }^{241}$ PU DECAY CHAIN

\begin{tabular}{|c|c|c|c|c|c|c|c|c|}
\hline $\begin{array}{l}\text { Time } \\
(y r) \\
\end{array}$ & $\begin{array}{l}2+1 \mathrm{Pu} \\
(\times 1) \\
\end{array}$ & & $\begin{array}{l}2 \div i \mathrm{Am} \\
\left(\times 10^{-3}\right) \\
\end{array}$ & $\begin{array}{l}2{ }^{37} \mathrm{~Np} \\
\left(\times 10^{-8}\right) \\
\end{array}$ & $\begin{array}{l}=3 \therefore \mathrm{Pa} \\
\left(\times 10^{-9}\right) \\
\end{array}$ & $\begin{array}{l}230 \\
\left(\times 10^{-12}\right) \\
\end{array}$ & $\begin{array}{l}22 . \mathrm{Th} \\
\left(\times 10^{-2}-4\right) \\
\end{array}$ & $\begin{array}{l}.25 \mathrm{Ra} \\
\left(10^{-}\right) \\
\end{array}$ \\
\hline 0.5 & 0.9762 & & 0.7869 & 0.0640 & 0.0425 & & & \\
\hline 1 & 0.9529 & & $1.55 \%$ & 0.2538 & 0.2057 & 0.00034 & & \\
\hline 2 & 0.9080 & & 3.033 & 0.9988 & 0.8993 & 0.00244 & & \\
\hline 4 & 0.8245 & & 5.778 & 3.867 & 3.673 & 0.02072 & & \\
\hline 6 & $0.7 \div 87$ & & 8.260 & 8.428 & 8.147 & 0.07007 & & \\
\hline 8 & 0.6798 & & 10.50 & 14.52 & 14.16 & 0.1645 & & \\
\hline 10 & 0.6173 & & 12.33 & 21.99 & 21.56 & 0.3163 & & \\
\hline 12 & 0.5606 & & 14.37 & 30.71 & 30.22 & 0.5373 & & \\
\hline 14 & 0.5090 & & 16.02 & 40.56 & 40.01 & 0.8372 & & \\
\hline 16 & 0.4622 & & 17.51 & 51.43 & 50.83 & 1.225 & 0.0419 & 0.00 it \\
\hline 18 & $0.419 \%$ & & 18.85 & 63.22 & 62.57 & 1.710 & 0.0656 & $0.01: 7$ \\
\hline 20 & 0.3811 & & 20.07 & 75.83 & 75.17 & 2.298 & 0.1004 & $0.059 j$ \\
\hline 22 & 0.3460 & & 21.16 & 89.20 & 88.47 & 2.998 & 0.1459 & 0.0961 \\
\hline 24 & 0.3142 & & 22.15 & 103.2 & 102.5 & 3.814 & 0.2062 & 0.1542 \\
\hline 30 & 0.2353 & & 24.53 & 148.7 & 147.9 & 7.017 & 0.5008 & 0.4510 \\
\hline 40 & 0.1452 & & 27.09 & 232.7 & 231.8 & 15.11 & 1.524 & 1.468 \\
\hline 50 & 8.965 & $\times 10^{-2}$ & 28.48 & 322.9 & 322.0 & 26.94 & 3.459 & 3.394 \\
\hline 60 & 5.535 & $\therefore \quad 10^{-\therefore}$ & 29.16 & 416.5 & 415.5 & +2.71 & 6.763 & 6.699 \\
\hline 70 & 3.417 & $10^{--}$ & 29.39 & 511.4 & 510.4 & 62.51 & 11.66 & 11.58 \\
\hline 80 & 2.109 & $\times 10^{-2}$ & 29.35 & 606.6 & 605.5 & 86.38 & 18.65 & 18.56 \\
\hline 90 & 1302 & $\because 10=$ & 29.15 & 701.3 & 700.3 & 111.4 & 28.04 & 27.93 \\
\hline 100 & 8.038 & $\times 10$ & 28.86 & 795.3 & 794.3 & 146.3 & 40.26 & 40.14 \\
\hline 200 & 6.461 & $<10^{-3}$ & 24.84 & 1666 & 1665 & 676.9 & 398.0 & 397.6 \\
\hline 400 & 4.174 & $\times 10^{-y}$ & 18.06 & 3043 & 3043 & 2721 & 3392 & 3391 \\
\hline 600 & 2.697 & $\times 10^{-1}=$ & 13.13 & 4045 & 4045 & 5773 & 11150 & 11150 \\
\hline 800 & 1.743 & $\times 10^{-17}$ & 9.549 & 4773 & 4773 & 9556 & 25190 & 25190 \\
\hline 1000 & 1.126 & $\times 10^{-2} 1$ & 6.943 & 5303 & 5302 & 13870 & 46580 & 46570 \\
\hline
\end{tabular}




\begin{tabular}{|c|c|c|c|c|c|c|c|}
\hline $\begin{array}{l}\text { Time } \\
(\mathrm{yr})\end{array}$ & $\begin{array}{l}225 \mathrm{Ac} \\
\left(\times 10^{-14}\right) \\
\end{array}$ & $\begin{array}{l}\text { Eal } \mathrm{Fr} \\
\left(\times 10^{-14}\right) \\
\end{array}$ & $\begin{array}{l}217 \mathrm{At} \\
\left(\times 10^{-14}\right) \\
\end{array}$ & $\begin{array}{l}213 \mathrm{Bi} \\
\left(\times 10^{-14}\right) \\
\end{array}$ & $\begin{array}{l}213 \mathrm{Po} \\
\left(\times 10^{-14}\right) \\
\end{array}$ & $\begin{array}{l}20, \mathrm{~T} 1 \\
\left(\times 10^{-14}\right) \\
\end{array}$ & $\begin{array}{l}209 \mathrm{~Pb} \\
\left(\times 10^{-14}\right) \\
\end{array}$ \\
\hline $\begin{array}{l}0.5 \\
1 \\
2 \\
4 \\
6 \\
8 \\
10 \\
12 \\
14\end{array}$ & & & & & & & \\
\hline 16 & 0.0109 & 0.06960 & 0.07427 & 0.07847 & 0.07571 & 0.001684 & 0.02951 \\
\hline 18 & 0.0340 & 0.09384 & 0.09926 & 0.1032 & 0.09905 & 0.002200 & 0.05460 \\
\hline 20 & 0.0686 & 0.1268 & 0.1315 & 0.1401 & 0.1339 & U 002960 & 0.08688 \\
\hline 22 & 0.1078 & 0.1702 & 0.1739 & 0.1795 & 0.1753 & 0.003847 & $0 \quad 1360$ \\
\hline 24 & 0.1630 & 0.2311 & 02356 & 0.2415 & 0.2360 & $0 \cap 05192$ & 0.918 \\
\hline 30 & 0.4523 & 0.5220 & 0.5258 & 0.5315 & 0.5186 & $0.0116 ?$ & 0.4818 \\
\hline 40 & 1.479 & 1.538 & 1.544 & 1.550 & 1.514 & 0.03397 & 1.501 \\
\hline 50 & 3.397 & 3.463 & 3.467 & 3.473 & 3.393 & 0.07633 & 3.420 \\
\hline 60 & 6.697 & 6.754 & 6.759 & 6.763 & 6.612 & C. 1487 & 6.711 \\
\hline 70 & 11.57 & 11.63 & 11.64 & 11.64 & 11.39 & 0.2561 & 11.59 \\
\hline 80 & 18.53 & 18.60 & 18.61 & 18.61 & 18.20 & 0.4093 & 18.56 \\
\hline 90 & 27.90 & 27.96 & 27.97 & 27.97 & 27.36 & 06153 & 27.92 \\
\hline 100 & 40.10 & 40.15 & 40.16 & 40.16 & 39.28 & 0.8835 & 40.11 \\
\hline 200 & 397.4 & 397.4 & 397.5 & 397.5 & 338.7 & 8.744 & 397.4 \\
\hline 400 & 3390 & 3390 & 3390 & 3390 & 3315 & 74.57 & 3390 \\
\hline 600 & 11140 & 11150 & 11150 & 11150 & 10900 & $\left.2 I_{r}\right) .2$ & 11150 \\
\hline 800 & 25190 & 25190 & 25190 & 25190 & 24630 & 554.1 & 25190 \\
\hline 1000 & 46570 & 46570 & 46570 & 46570 & 45540 & 1024 & 46570 \\
\hline
\end{tabular}


Table 12

RELATIVE NUMBER OF ATOMS FOR MEMBERS OF THE ${ }^{24}{ }^{2} \mathrm{Pu}$ DECAY CHAIN

\begin{tabular}{|c|c|c|c|c|c|}
\hline $\begin{array}{l}\text { Time } \\
(\mathrm{yr})\end{array}$ & 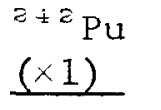 & $\begin{array}{c}2{ }^{38} \mathrm{U} \\
\left(\times 10^{-E}\right) \\
\end{array}$ & $\begin{array}{l}23^{4} \mathrm{Th} \\
\left(\times 10^{-15}\right)\end{array}$ & $\begin{array}{r}234 \mathrm{~Pa} \\
\left(\times 10^{-20}\right) \\
\end{array}$ & $\begin{array}{l}234 \mathrm{U} \\
\left(\times 10^{-13}\right) \\
\end{array}$ \\
\hline 0.5 & 1.0000 & .0896 & .0106 & .0360 & \\
\hline 1 & 1.0000 & .1791 & .0237 & .0804 & \\
\hline 2 & 1.0000 & .3582 & .0499 & .1693 & \\
\hline 4 & 1.0000 & .7164 & .1023 & .3470 & .071 \\
\hline 6 & 1.0000 & 1.075 & .1547 & .5248 & .136 \\
\hline 8 & 1.0000 & 1.433 & .2071 & .7025 & .133 \\
\hline 10 & 1.0000 & 1.791 & .2595 & .8803 & .169 \\
\hline 12 & 1.0000 & 2.149 & .3119 & 1.058 & .256 \\
\hline 14 & 1.0000 & 2.507 & .3644 & 1.236 & .330 \\
\hline 16 & 1.0000 & 2.866 & .4168 & 1.413 & .444 \\
\hline 18 & 1.0000 & 3.224 & .4692 & 1.591 & .480 \\
\hline 20 & 1.0000 & 3.582 & .5216 & 1.769 & .539 \\
\hline 22 & 1.0000 & 3.940 & .5740 & 1.947 & .669 \\
\hline 24 & 1.0000 & 4.298 & .6264 & 2.124 & .793 \\
\hline 30 & .9999 & 5.373 & .7836 & 2.658 & 1.351 \\
\hline 40 & .9999 & 7.164 & 1.046 & 3.546 & 2.292 \\
\hline 50 & .9999 & 8.955 & 1.308 & 4.435 & 3.522 \\
\hline 60 & .9999 & 10.75 & 1.570 & 5.324 & 4.989 \\
\hline 70 & .9999 & 12.54 & 1.832 & 6.212 & 6.748 \\
\hline 80 & .9999 & 14.33 & 2.094 & 7.101 & 8.836 \\
\hline 90 & .9998 & 16.12 & 2.356 & 7.989 & 11.19 \\
\hline 100 & .9998 & 17.91 & 2.618 & 8.878 & 13.78 \\
\hline 200 & .9996 & 35.82 & 5.237 & 17.76 & 55.04 \\
\hline 400 & .9993 & 71.62 & 10.48 & 35.53 & 220.0 \\
\hline 600 & .9989 & 107.4 & 15.71 & 53.29 & 494.8 \\
\hline 800 & .9986 & 143.2 & 20.95 & 71.04 & 879.6 \\
\hline 1000 & .9982 & 178.9 & 26.18 & 88.78 & 1373 \\
\hline
\end{tabular}


RELATIVE ACTIVITY FOR MEMBERS OF THE ${ }^{242}$ Pu DECAY CHAIN

\begin{tabular}{|c|c|c|c|c|c|}
\hline $\begin{array}{l}\text { Time } \\
(y r)\end{array}$ & $\begin{array}{l}24^{2} \mathrm{Pu} \\
(\times 1) \\
\end{array}$ & $\begin{array}{l}{ }^{23} \mathrm{U} \\
\left(\times 10^{-15}\right) \\
\end{array}$ & $\begin{array}{r}234 \mathrm{Th} \\
\left(\times 10^{-15}\right) \\
\end{array}$ & $\begin{array}{l}234 \mathrm{~Pa} \\
\left(\times 10^{-1 E}\right) \\
\end{array}$ & $\begin{array}{l}234 \mathrm{U} \\
\left(\times 10^{-13}\right) \\
\end{array}$ \\
\hline 0.5 & 1.0000 & .1376 & .1116 & .1116 & \\
\hline 1 & 1.0000 & .2753 & .2491 & .2491 & \\
\hline 2 & 1.0000 & .5505 & .5243 & .5244 & \\
\hline 4 & 1.0000 & 1.101 & 1.075 & 1.075 & .1992 \\
\hline 6 & 1.0000 & 1.652 & 1.625 & 1.625 & .3817 \\
\hline 8 & 1.0000 & 2.202 & 2.175 & 2.176 & .3732 \\
\hline 10 & 1.0000 & 2.753 & 2.726 & 2.726 & .4743 \\
\hline 12 & 1.0000 & 3.303 & 3.277 & 3.277 & .7184 \\
\hline 14 & 1.0000 & 3.854 & 3.828 & 3.827 & .9261 \\
\hline 16 & 1.0000 & 4.404 & 4.378 & 4.378 & 1.246 \\
\hline 18 & 1.0000 & 4.955 & 4.929 & 4.929 & 1.267 \\
\hline 20 & 1.0000 & 5.505 & 5.479 & 5.479 & 1.513 \\
\hline 22 & 1.0000 & 6.055 & 6.030 & 6.030 & 1.877 \\
\hline 24 & 1.0000 & 6.606 & 6.580 & 6.580 & 2.225 \\
\hline 30 & .9999 & 8.258 & 8.232 & 8.232 & 3.791 \\
\hline 40 & .9999 & 11.01 & 10.98 & 10.98 & 6.432 \\
\hline 50 & .9999 & 13.76 & 13.74 & 13.74 & 9.884 \\
\hline 60 & .9999 & 16.51 & 16.49 & 16.49 & 14.00 \\
\hline 70 & .9999 & 19.27 & 19.24 & 19.24 & 18.94 \\
\hline 80 & .9999 & 22.02 & 21.99 & 21.99 & 24.80 \\
\hline 90 & .9998 & 24.77 & 24.75 & 24.75 & 31.39 \\
\hline 100 & .9998 & 27.52 & 27.50 & 27.50 & 38.67 \\
\hline 200 & .9996 & 55.05 & 55.01 & 55.01 & 154.5 \\
\hline 400 & .9993 & 110.1 & 110.0 & 110.0 & 617.5 \\
\hline 600 & .9989 & 165.1 & 165.1 & 165.1 & 1389 \\
\hline 800 & .9986 & 220.1 & 220.0 & 220.0 & 2468 \\
\hline 1000 & .9982 & 275.0 & 275.0 & 275.0 & 3856 \\
\hline
\end{tabular}




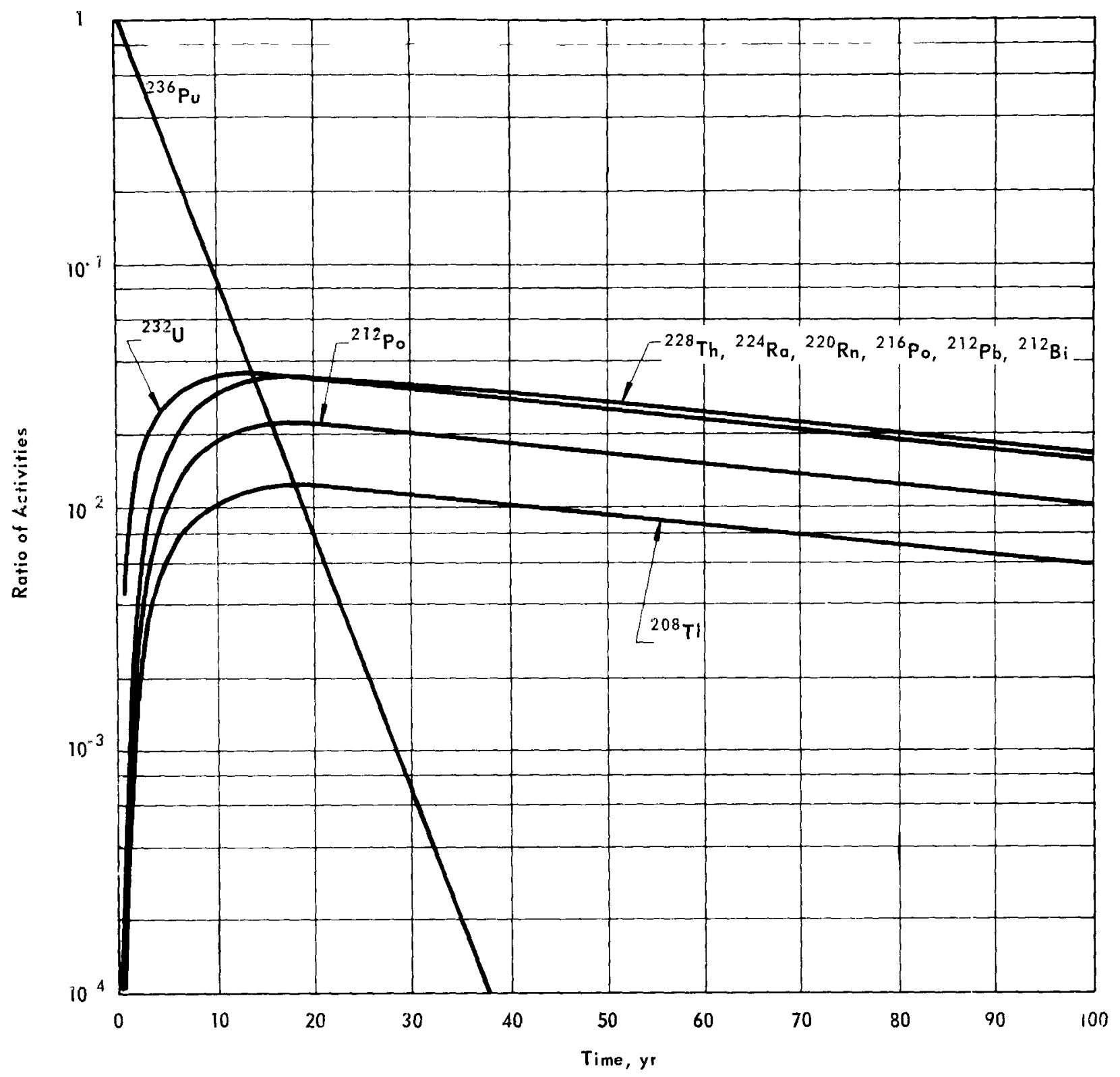

FIGURE 7 - Plot of the relative activities of the members of the ${ }^{236} \mathrm{Pu}$
decay chain. 


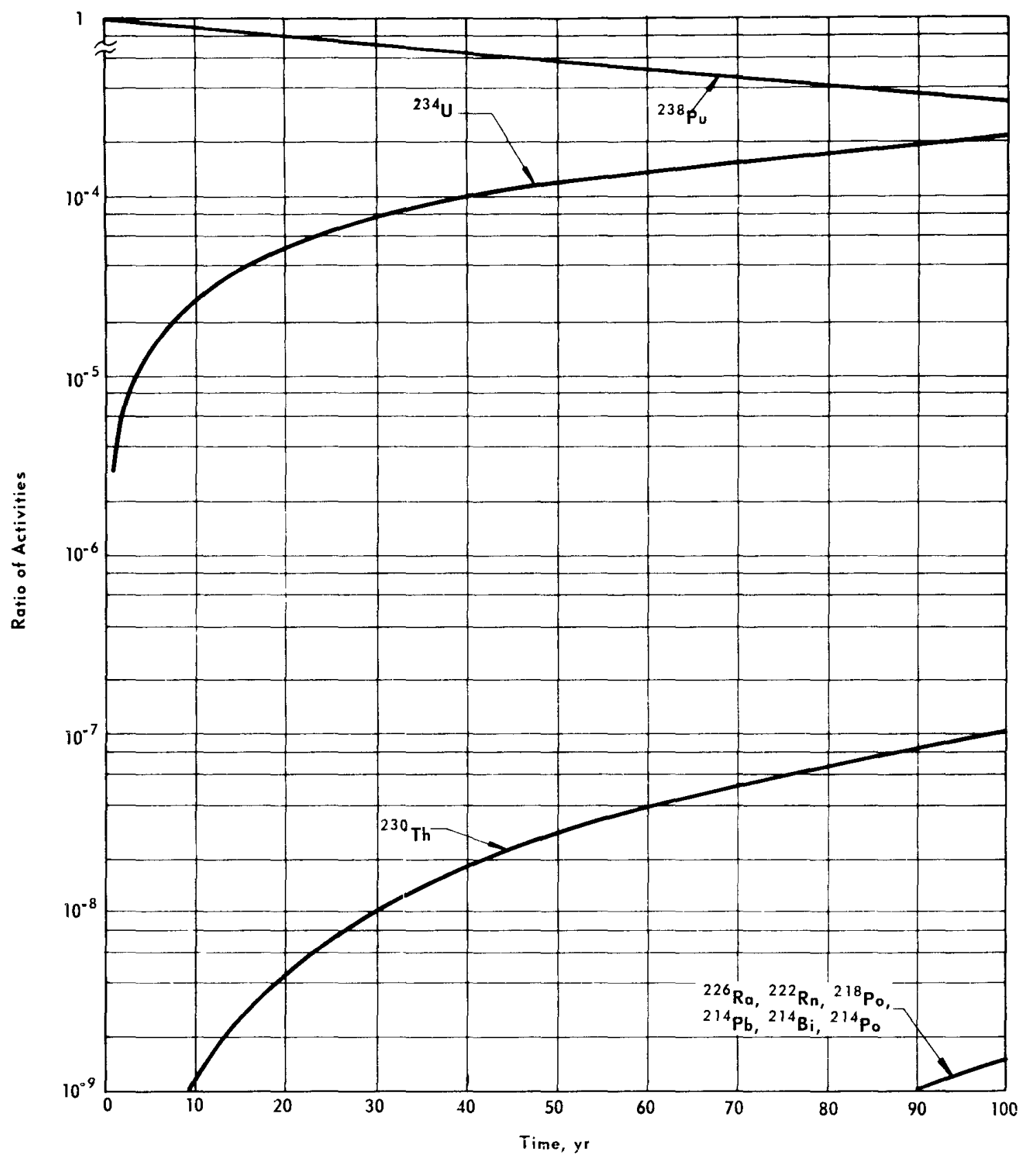

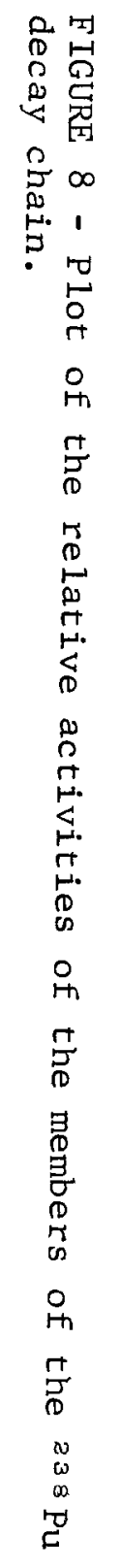




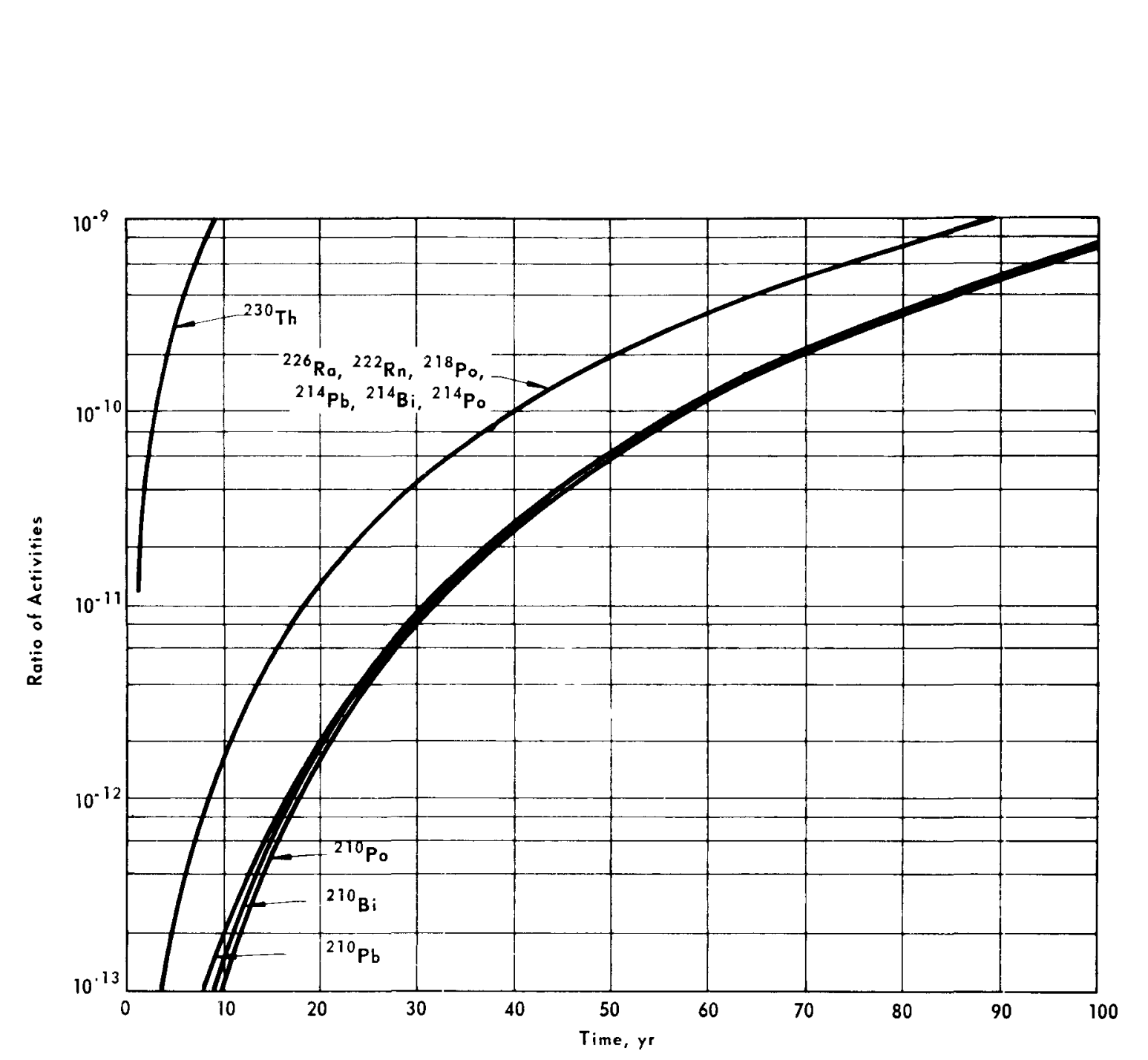

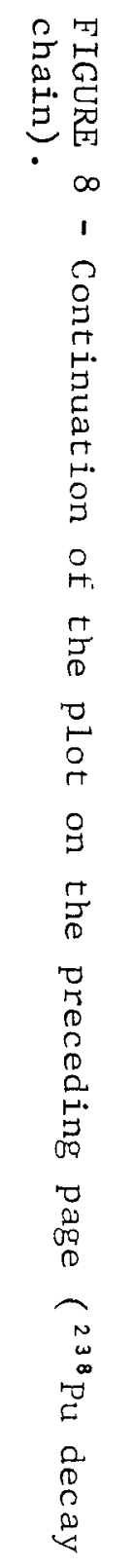




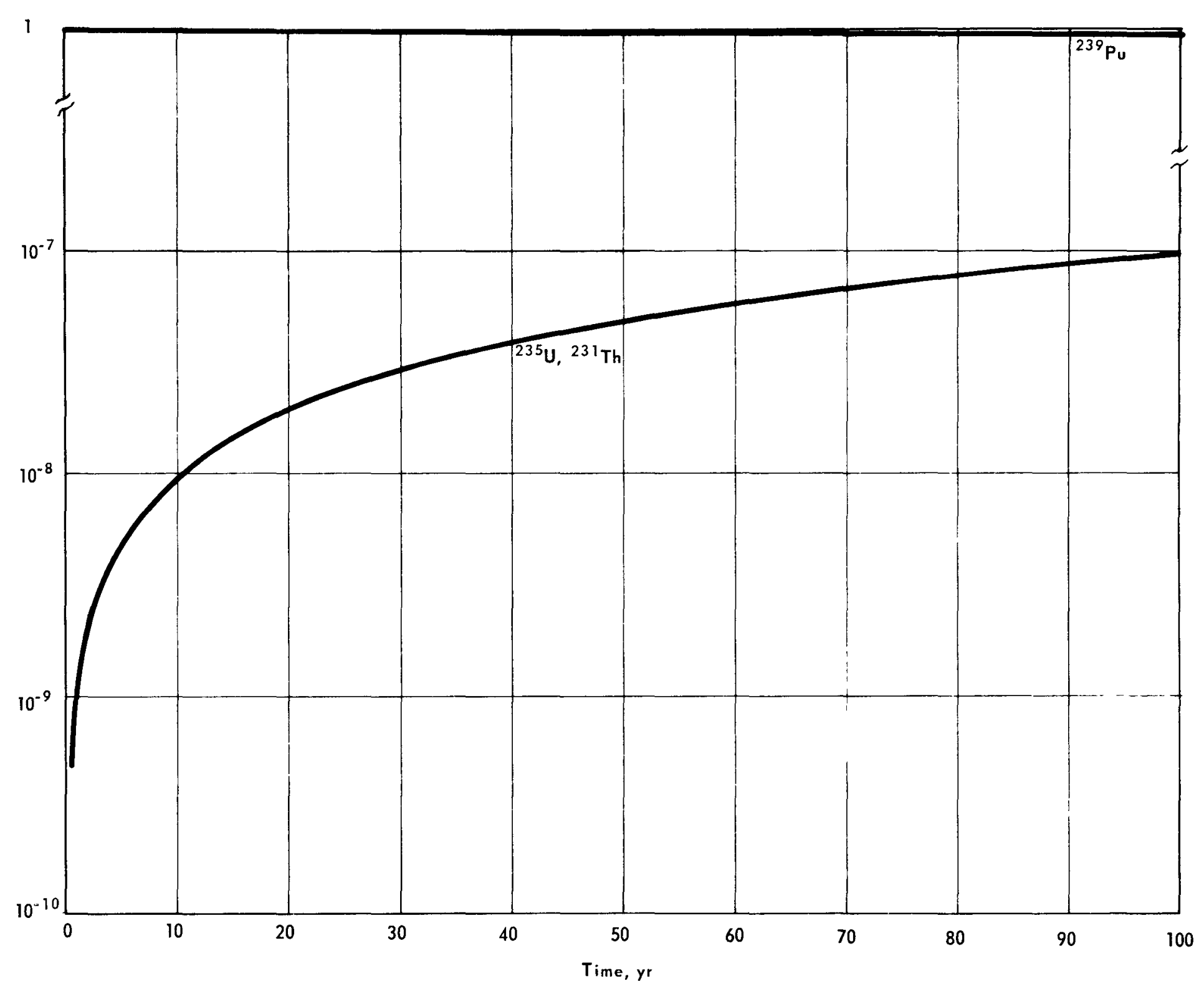

另王

مै

两

?

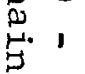

-

음

容

范

$\stackrel{\stackrel{0}{0}}{2}$

范

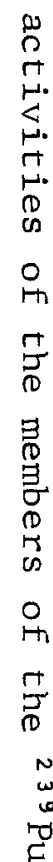

C. 


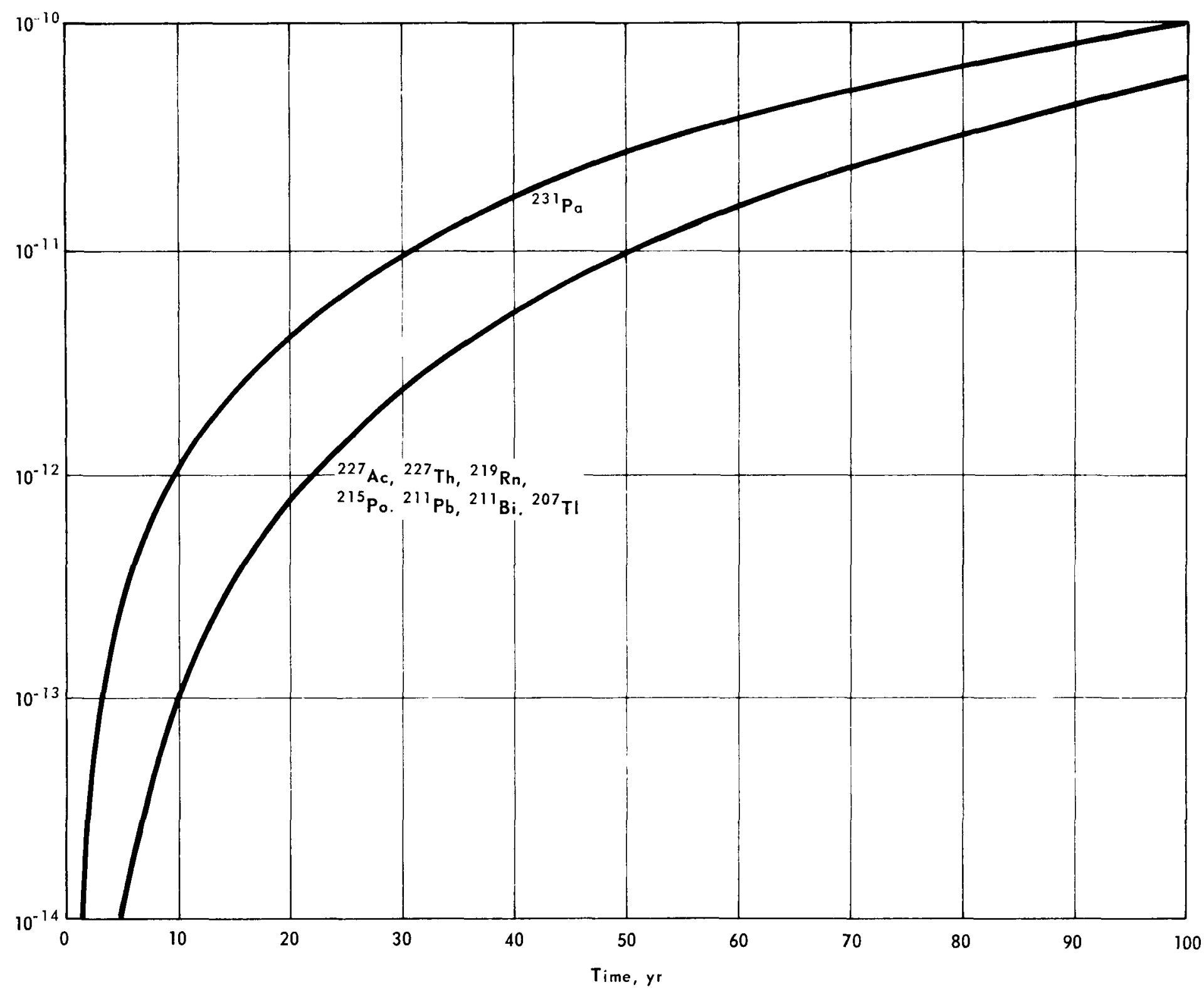

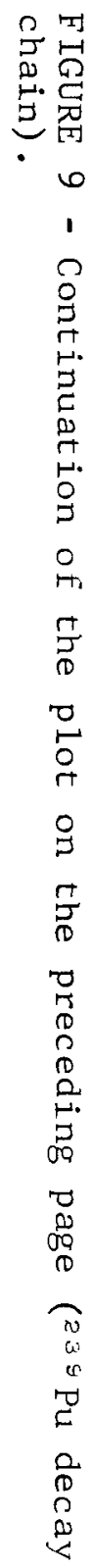




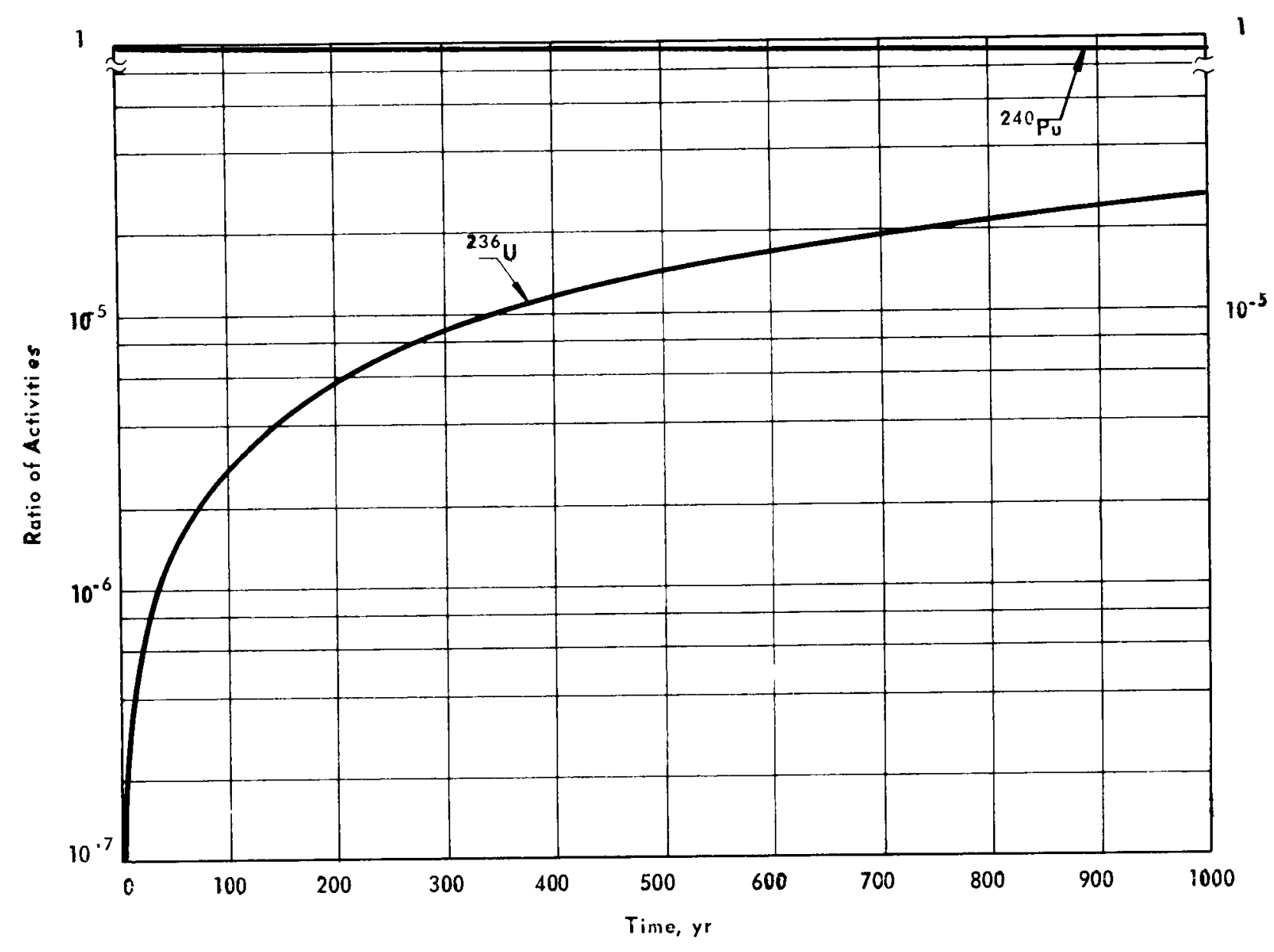

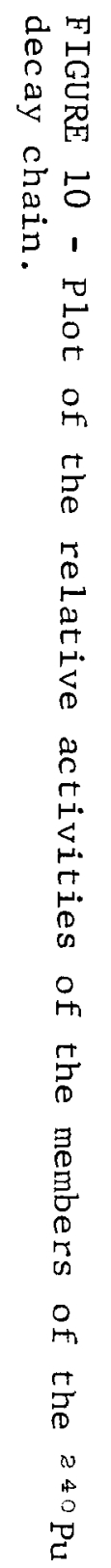




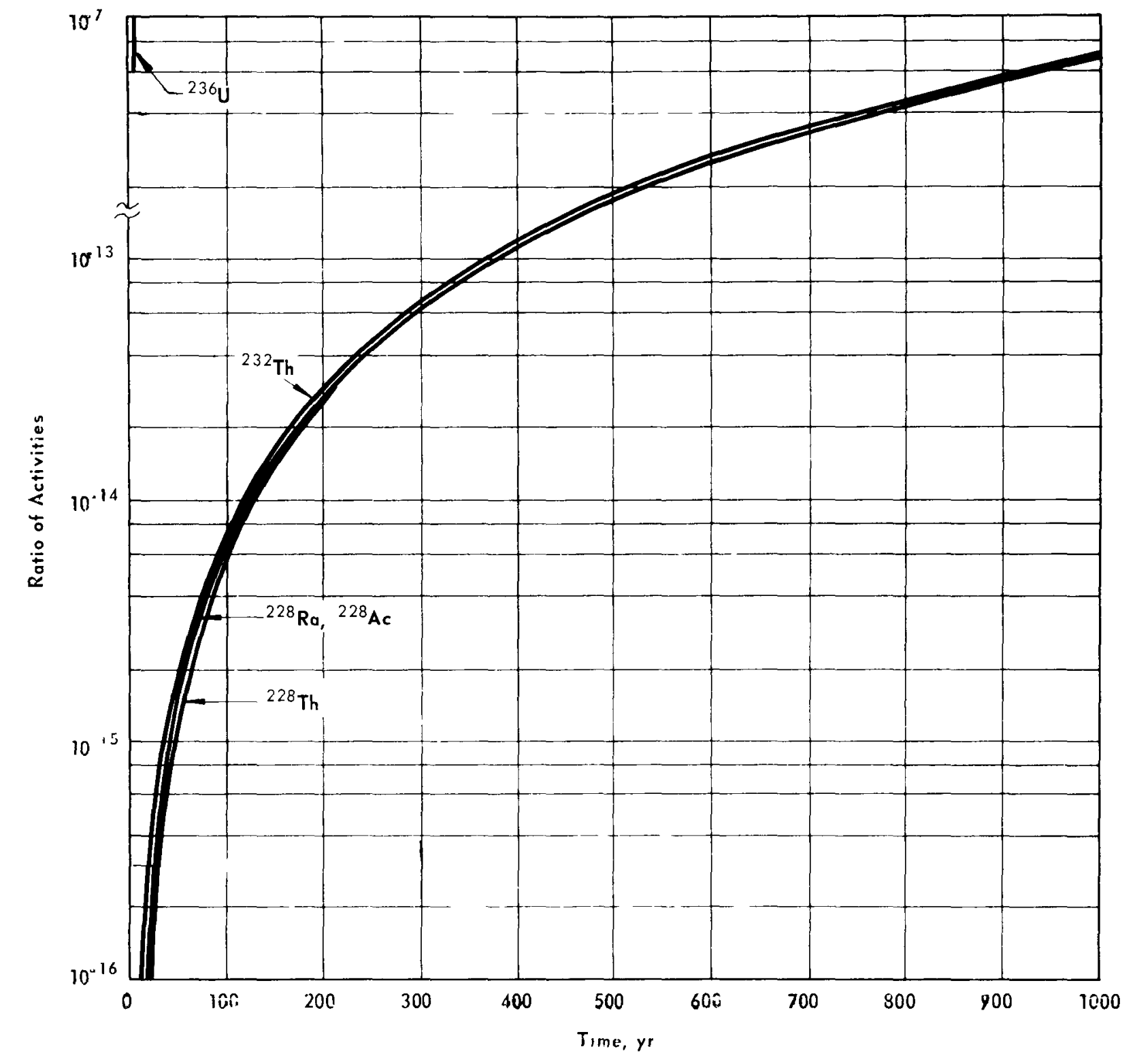

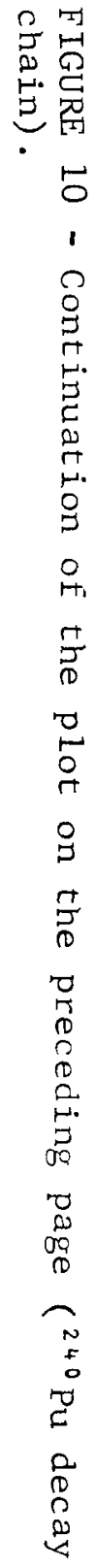


$\omega$

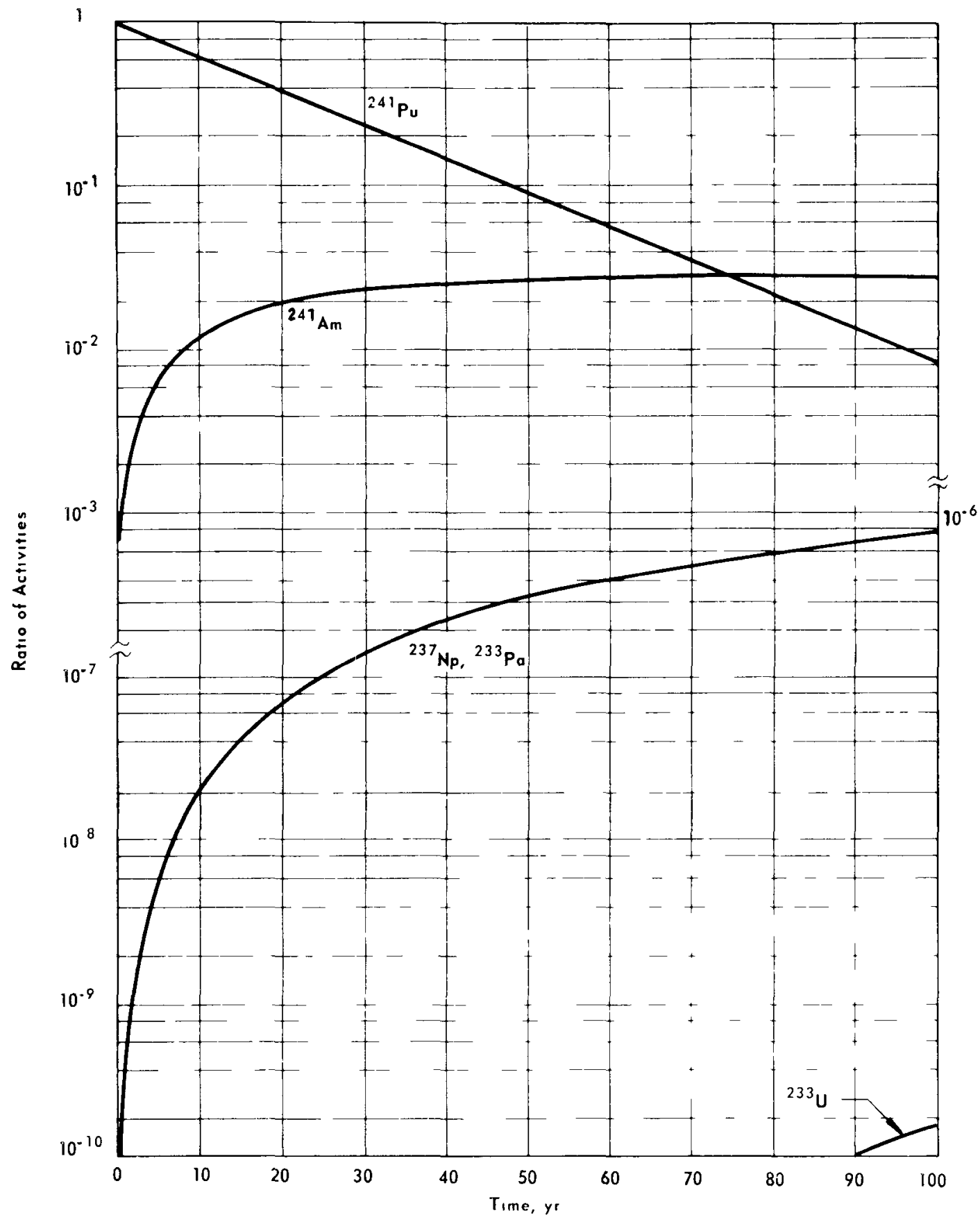

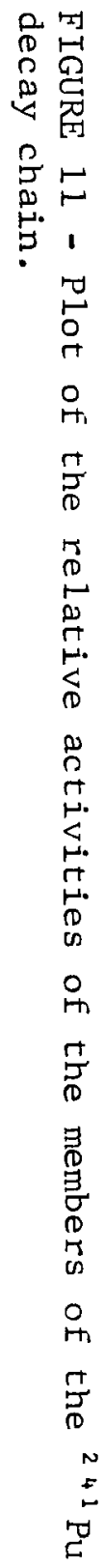

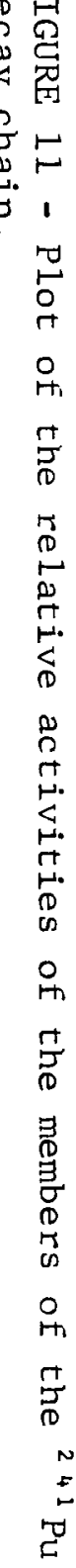




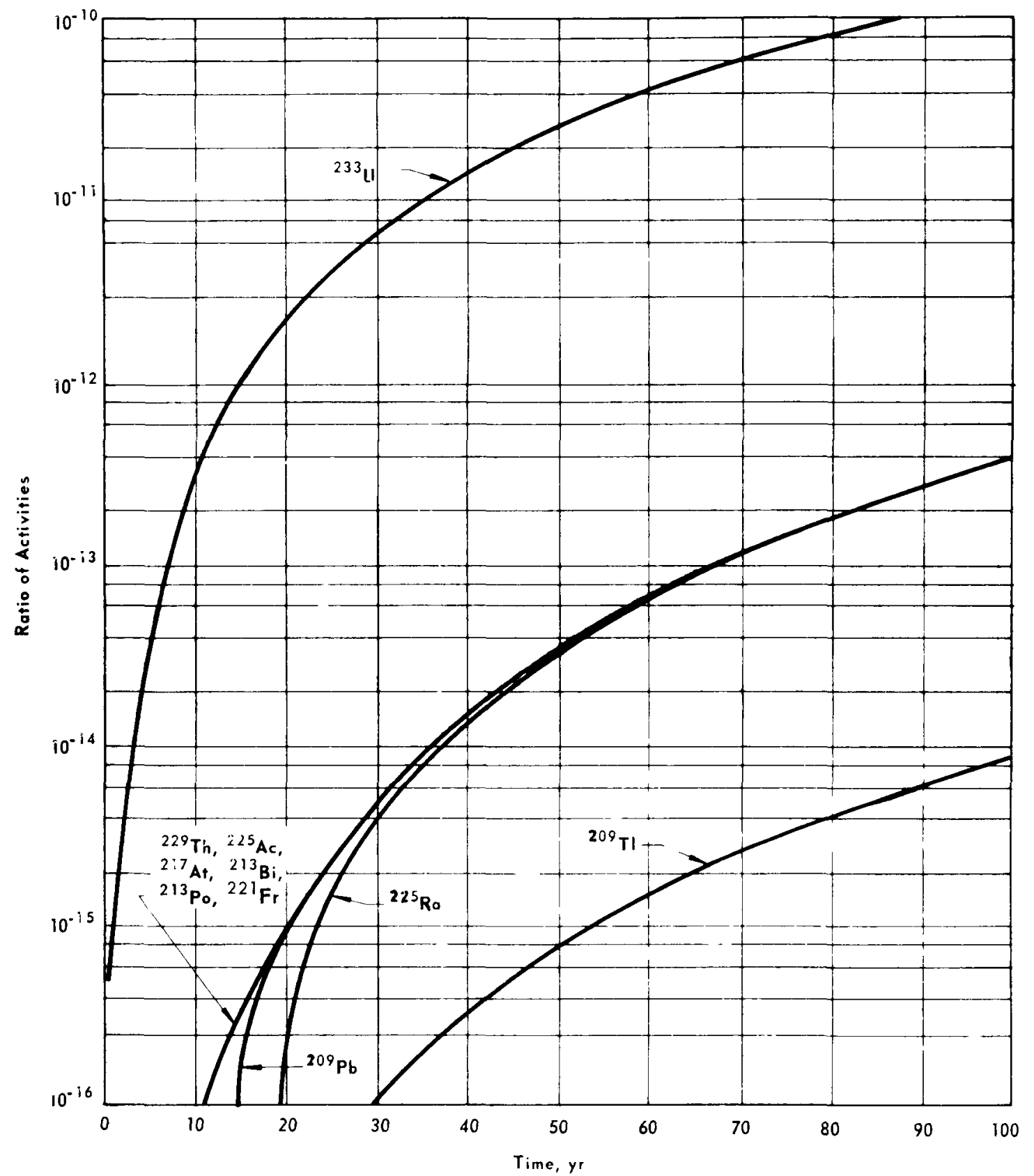

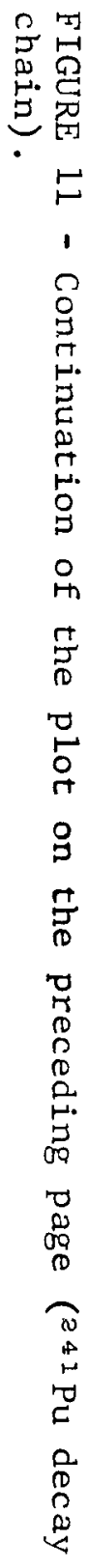




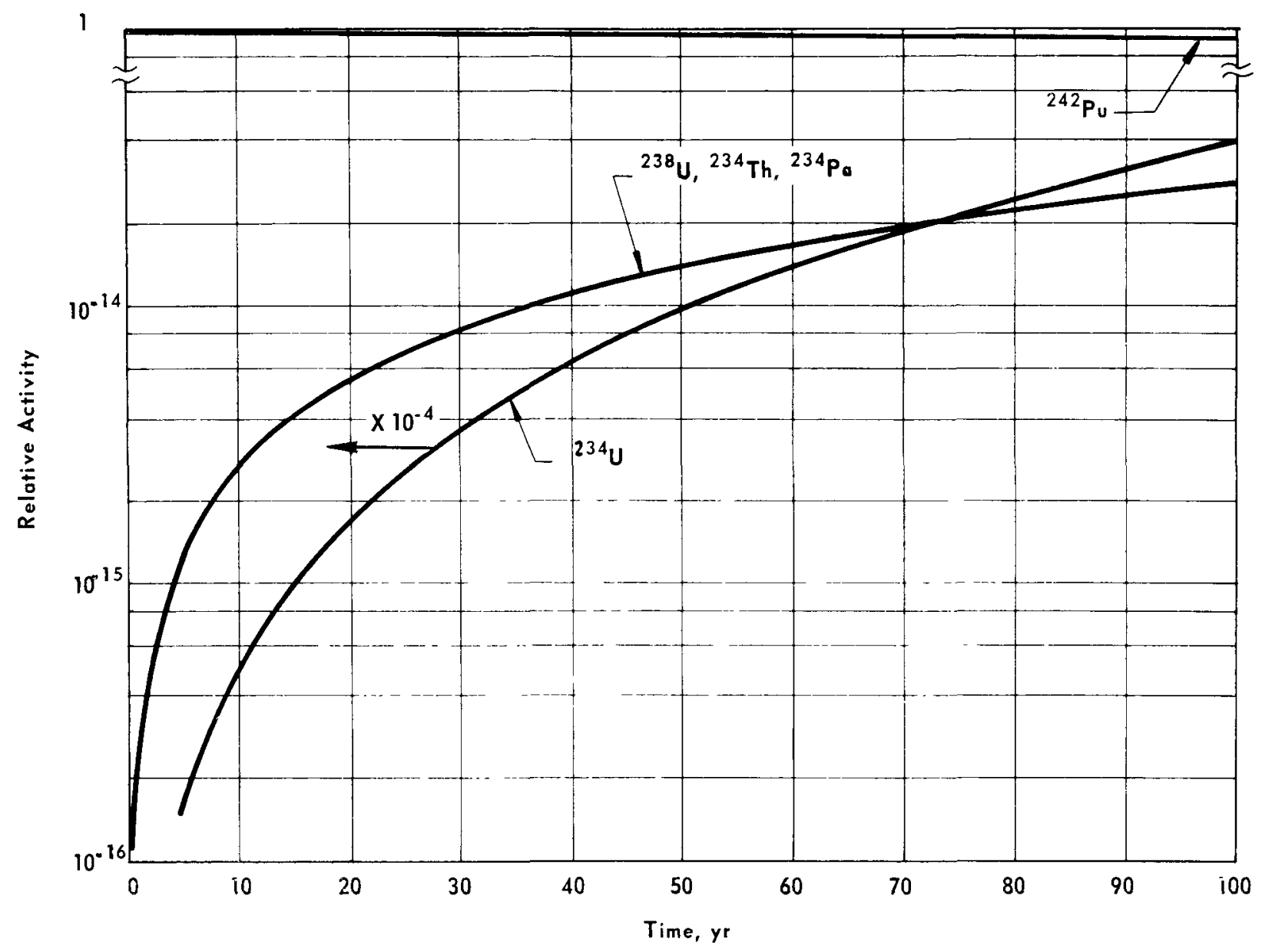

FIGURE 12 - Plot of the relative activities of the members of the ${ }^{242} \mathrm{Pu}$ decay chain. 


\section{$\underline{\text { References }}$}

1. "Plutonium-238 Isotopic Fue1 Form Data Sheets", MLM-1691, (October 31, 1969).

2. C. M. Lederer, J. M. Hollander, and I. Perlman, Table of Isotopes, John Wiley \& Sons, Inc., (1967).

3. H. Bateman, Proc. Cam. Phi1. Soc. 16, 423 (1910).

4. R. Sher, "Half-lives of ${ }^{3} \mathrm{H}$ and $2{ }^{3}{ }^{8} \mathrm{Pu}$ ", BNL 50233 ( $\mathrm{T}-570$ ), (April 1, 1970). 